ACCEPTED IN APJ

Preprint typeset using $\mathrm{LAT}_{\mathrm{EX}}$ style emulateapj v. 26/01/00

\title{
SIMULTANEOUS MULTIWAVELENGTH OBSERVATIONS OF THE LOW/HARD STATE OF THE X-RAY TRANSIENT SOURCE SWIFT J1753.5-0127
}

\author{
M. Cadolle Bel ${ }^{1,2,3}$, M. Ribó ${ }^{1,4,5}$, J. Rodriguez ${ }^{1,4}$, S. Chaty ${ }^{1,4}$, S. Corbel ${ }^{1,4}$, A.

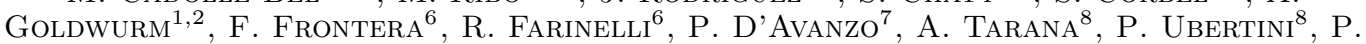 \\ LAURENT $^{1,2}$, P. Goldoni ${ }^{1,2}$ AND I. F. Mirabel ${ }^{9}$ \\ Accepted in ApJ
}

\begin{abstract}
We report the results of simultaneous multiwavelength observations of the X-ray transient source SWIFT J1753.5-0127 performed with INTEGRAL, RXTE, NTT, REM and VLA on 2005 August 10-12. The source, which underwent an X-ray outburst since 2005 May 30, was observed during the INTEGRAL Target of Opportunity program dedicated to new X-ray novae located in the Galactic Halo. Broad-band spectra and fast timing variability properties of SWIFT J1753.5-0127 are analyzed together with the optical, near infra-red and radio data. We show that the source was significantly detected up to $600 \mathrm{keV}$ with Comptonization parameters and timing properties typical of the so-called Low/Hard State of black hole candidates. We build a spectral energy distribution and we show that SWIFT J1753.5-0127 does not follow the usual radio/X-ray correlation of X-ray binaries in the Low/Hard State. We give estimates of distance and mass. We conclude that SWIFT J1753.5-0127 belongs to the X-ray nova class and that it is likely a black hole candidate transient source of the Galactic Halo which remained in the Low/Hard State during its main outburst. We discuss our results within the context of Comptonization and jet models.
\end{abstract}

Subject headings: black hole physics - stars: individual: SWIFT J1753.5-0127 - gamma rays: observations - X-rays: binaries - X-rays: general - optical: general - infra-red: binaries - radio continuum: general

1. INTRODUCTION

X-ray Novae (XN), also known as Soft X-ray Transients, are Low-Mass accreting X-ray Binaries (LMXB) usually in quiescent state. They may undergo sudden and bright few-month-long X-ray outbursts with typical recurrence periods of many years (Tanaka \& Shibazaki 1996). Most of the XN are believed to harbor a Black Hole (BH) as the compact object. Because of large changes in the effective accretion rate during the outburst, $\mathrm{XN}$ often pass through some of the spectral states that have been identified in $\mathrm{BH}$ binaries (e.g., McClintock \& Remillard 2006). This gives us the possibility to study the physical properties of emitting regions (disk, corona, jets) and their evolutions. The two main spectral states are the Thermal Dominant State (TDS) and the Low/Hard State (LHS). They are characterized by different combinations of the soft (multicolor black-body coming from an optically thick and geometrically thin accretion disk), hard (power law, with a possible break at 50-100 keV) and reflection (continuum excess, fluorescence lines and K-edge) spectral components. These characteristics are coupled to different properties of variability, to Quasi Periodic Oscillations (QPOs) observed in the power spectrum (e.g., Belloni 2001, 2005) and to radio changes. Indeed, a compact jet is usually observed in the
LHS while it is quenched in the TDS (Corbel et al. 2000, 2003; Gallo et al. 2003, 2006). The jet can contribute to the high-energy emission and models where the base of a compact jet plays a major role have been proposed (Markoff et al. 2005).

At the onset of an XN outburst, the hard X-rays usually reach the maximum first; after that, a soft component develops. Then, BH XN usually display the TDS characterized by a power law (with $\Gamma \sim 2.5$ ) extending to high energies along with the strong ultra-soft component. Some sources tend to evolve into the LHS in the late stages of the outburst (e.g., Cadolle Bel et al. 2004). The spectrum is then dominated by a hard power law $(\Gamma \sim 1.5-2.0)$ with a break, attributed to the Comptonization of the low energy radiation by a hot electron cloud (or corona/jet) with thermal distribution. The soft component, probably emitted by the disk, is very weak. In the past, distinct states have been studied by combining data from several high-energy instruments (e.g. Grove et al. 1998; Zdziarski et al. 2001; Rodriguez et al. 2003; Cadolle Bel et al. 2006). Larger samples of simultaneous observations over an extended energy range are necessary: they may help us to better understand the relation between the different com-

\footnotetext{
${ }^{1}$ Service d'Astrophysique, DSM/DAPNIA/SAp, CEA-Saclay, 91191 Gif-sur-Yvette, France

${ }^{2}$ AstroParticule et Cosmologie, UMR 7164, (CEA/CNRS/Université Paris 7/Observatoire de Paris), France

${ }^{3}$ European Space Astronomy Centre (ESAC), Madrid (Spain); Marion.Cadolle@sciops.esa.int

${ }^{4}$ Astrophysique Intéractions Multi-échelles, UMR 7158 (CEA/CNRS/Université Paris 7), France

${ }^{5}$ Universitat de Barcelona, Spain

${ }^{6}$ TESRE/CNR, Bologna \& Università di Ferrara, Italy

${ }^{7}$ INAF, Osservatorio Astronomico di Brera, Merate \& Università degli Studi dell'Insubria, Como, Italy

${ }^{8} \mathrm{IASF} / \mathrm{INAF}$ Roma \& Università di Roma Tor Vergata, Italy

${ }^{9} \mathrm{ESO}$, Santiago, Chile
} 
ponents, their links with the changes in the accretion rate and the nature of any additional parameter that may drive the state changes (e.g., Homan et al. 2001; Rodriguez et al. 2003). In the recent years, many BH Candidates (BHC) have undergone outbursts with no transitions, e.g., GS 1354-64 (Brocksopp et al. 2001) or XTE J1118+480 (Remillard et al. 2005). Even some neutron stars did so (like Aquila X-1 in Rodriguez et al. 2006a): a systematic study of those systems may reveal important clues to understand the physical mechanisms of an XN outburst.

SWIFT J1753.5-0127 was discovered in hard X-rays with the Swift/Burst Alert Telescope (BAT) on 2005 May 30 (Palmer et al. 2005). The Swift/X-ray Telescope (XRT) observation revealed a variable source with a spectrum well fitted by a power law of photon index $\Gamma \sim 2.1$ and with a column density $N_{\mathrm{H}} \sim 2.0 \times 10^{21}$ atoms $\mathrm{cm}^{-2}$ (Morris et al. 2005). This latter value is compatible with a location of SWIFT J1753.5-0127 at several kpc without requiring intrinsic absorption. The source was also clearly detected in UV with the Swift/UV Optical Telescope UVOT (Still et al. 2005). On the ground, the optical counterpart found with the MDM $2.4 \mathrm{~m}$ telescope revealed a new star within the Swift error circle (Halpern et al. 2005). At the beginning of July, Fender et al. (2005) conducted a 8 hour radio observing run at a frequency of $1.7 \mathrm{GHz}(18 \mathrm{~cm}$ wavelength) with the Multi-Element Radio-Linked Interferometer Network (MERLIN). They reported a probable point-like radio counterpart which is consistent with a compact jet. In X-rays, the $1.2-12 \mathrm{keV}$ source flux increased to the maximum value of $\sim 200$ $\mathrm{mCrab}$ in few days (see Figure 1 at MJD 53560) and then it started to decay slowly. The hard power law spectrum observed with the Swift/XRT (Morris et al. 2005) and the $0.6 \mathrm{~Hz} \mathrm{QPO}$ detected in pointed RXTE observations are characteristic of the LHS (Morgan et al. 2005; Ramadevi \& Seetha 2006). Preliminary INTEGRAL observations lead to the same conclusion (Cadolle Bel et al. 2005). Seven months after the observations shown in this work (i.e., end of $2006 \mathrm{March}$ ), the same conclusion was reached by Miller et al. (2006) who observed the source up to $100 \mathrm{keV}$ when it was fainter.

According to the characteristics mentioned above, SWIFT J1753.5-0127 may be a good BHC and it was therefore an excellent target for the INTEGRAL mission. Consequently, we triggered our Target of Opportunity (ToO) campaign for XN in the Galactic Halo on August 10-12. This program targets XNs located in regions out of the galactic disk and bulge where they are particularly prone to be studied at different wavelengths because less subject to absorption and source confusion. Preliminary results reported in Cadolle Bel et al. (2005) showed that the source was then still in a LHS in the decay phase of the eruption. Thanks to the large multi-wavelength program associated to the INTEGRAL ToO observation, we could also trigger simultaneously optical (EMMI on NTT and ROSS on REM), Near Infra-Red (hereafter NIR) with REMIR (on REM) and radio (VLA) observations on August 11 for 3.6, 1, 7.3 and 16.2 ks exposure times respectively. Moreover, RXTE pointed observations ( $\sim 3 \mathrm{ks})$ taken during our ToOs were available so we added them in our analysis. We report here the results of our INTEGRAL ToO observations on SWIFT J1753.5-0127 together with $R X T E$ and optical/NIR/radio data, starting with the description of the available data and of the analysis procedures employed (Section 2). Our results are presented in Section 3 before their discussion and interpretations (Section 4).

\section{OBSERVATIONS AND DATA REDUCTION}

Table 1 reports the log of the performed observations of SWIFT J1753.5-0127 giving for each of them the instrument data available, dates, exposure (per instrument) and observing modes. The INTEGRAL observations were performed on 2005 August 10-12 with a HEX dither pattern (Jensen et al. 2003) consisting of series of seven pointings, one on the source and six around it at $2^{\circ}$ distance (along an hexagonal pattern). The period of our INTEGRAL observations is also indicated in Figure 1, it shows the $1.2-12 \mathrm{keV} R X T E / \mathrm{ASM}$ daily average light curve since the discovery of the XN up to 2006 June 15.

\subsection{INTEGRAL data analysis}

We reduced the IBIS and JEM-X data with the standard analysis procedures of the Off-Line Scientific Analysis OSA 5.1 released by ISDC, with algorithms described in Goldwurm et al. (2003) and Westergaard et al. (2003) for IBIS and JEM-X respectively. Only the JEM-X1 monitor operated during our observation. Following recommendations of the OSA 5.1 release, IBIS/ISGRI events were selected to have corrected energies $>15 \mathrm{keV}$ and rise time channels between 7 and 80 . For the background corrections, we used the set of IBIS/ISGRI (default) maps derived in 256 energy channels from empty field observations. For the off-axis correction, maps and response matrices, we used those of the official OSA 5.1 release; all the maps were rebinned to our chosen energy bins. We took the latest available response files of OSA 5.1 which are time dependent and based on recent Crab observations. In the analysis, we considered the presence of other sources which were active in the considered region. Apart from the Swift XN no source was detected above $80 \mathrm{keV}$; only the neutron star binary system $4 \mathrm{U} 1812-12$ was active above $40 \mathrm{keV}$. We extracted light curves and spectra in different energy bands between 17-20 and $500 \mathrm{keV}$ and we built light curves and Hardness Ratios (HR). The SPI data were preprocessed with OSA 5.1 using the standard energy calibration gain coefficients per orbit. The spiros 9.2 release (Skinner \& Connell 2003) was used to extract the spectra of SWIFT J1753.5-0127 with a background model proportional to the saturating event count rates in the Ge detectors. Concerning the instrumental response, versions 17 of the IRF (Image Response Files) and 4 of the RMF (Redistribution Matrix Files) were taken as our ToO took place after the failure of the two detectors.

\subsection{Rossi-XTE data analysis}

We reduced the $R X T E$ data using the LHEASOFT v6.0.4 package, following the standard methods described in the Cook Book in a way similar to what we have done in 
Rodriguez et al. (2003, 2006b). We defined the "basic" Good Time Intervals (GTI) as the times during which the offset pointing was less than $0.02^{\circ}$ from the source and the elevation above the Earth limb higher than $10^{\circ}$. We also required that GTI correspond to times when both the Proportional Counter Units (PCU) 0 and 2 were turned on. This resulted in $3104 \mathrm{~s}$ of good times.

We extracted Proportional Counter Array (PCA) spectra and $16 \mathrm{~s}$ light curves from standard 2 data of the top layers of PCU 0 and 2. We generated background spectra with pcabackest v3.0 using the calibration files available for bright sources. Response files were generated with pcarsp v10.1. Due to uncertainties in the PCA response, $0.6 \%$ systematics were added to all channels. In order to study the rapid temporal variability of SWIFT J1753.5-0127, we also extracted high resolution light curves from Event data with a time resolution around $500 \mu \mathrm{s}$. This allowed us to study the timing properties up to $\sim 1000 \mathrm{~Hz}$. We restricted the light curve extraction to absolute channels $5-121(2-52 \mathrm{keV})$ in order to limit effects of the background at low and high energies.

Finally, we extracted spectra from the High Energy Timing Experiment (HEXTE) in an identical way of that presented in Rodriguez et al. (2003): for each cluster (A and B), the "on" from the "off" source pointings were separated before extraction of source plus background and background spectra, that were further corrected for deadtime. Spectral responses were generated with hxtrsp v3.1.

\subsection{Optical and NIR data analysis}

Our ToO observations were carried out as part of the European Southern Observatory (ESO) program ID \#075.D0634 (PI S. Chaty). On 2005 August 11 (around U.T. 02) we obtained optical photometry in $B, V R$ and $I$ bands with the spectro-imager EMMI installed on the New Technology Telescope (NTT) at La Silla Observatory. We used the large field imaging of EMMI's detector which gives an image scale of $0^{\prime \prime} 166 \mathrm{pixel}^{-1}$ and a field of view of $9 ! 9 \times 9$ '0. Concerning the photometric observations, we took an integration time of $60 \mathrm{~s}$ for each exposure. We observed four photometric standard stars of the optical standard star catalog of Landolt et al. (1992): T Phe A, B, C and D. Rapid photometric observations were performed in the $V$ band during the time interval between MJD 53593.17-53593.24. They were simultaneous to the radio observations: 200 images of $1 \mathrm{~s}$ exposure time were obtained every $27 \mathrm{~s}$, covering a total of 93 minutes. We detected a scattering of $0.1 \mathrm{mag}$ (within the error bars) in the course of this rapid photometry. Further observations were performed with the REM telescope (Zerbi et al. 2001; Chincarini et al. 2003; Covino et al. 2004) equipped with the ROSS optical spectrograph/imager and the REMIR NIR camera. Observations were carried out on August 11.98, 17.99 and 27.04 (U.T.) in the optical ( $R$ filter) and on August 11.04 (U.T.) in the NIR (standard $J, H$ and $K_{s}$ filters). NIR photometric observations were calibrated against the $2 M A S S$ catalog.
We used the IRAF (Image Reduction and Analysis Facility) suite to perform data reduction, carrying out standard procedures of optical and NIR image reduction, including flat-fielding and subtraction of the blank NIR sky. We carried out aperture photometry and we then transformed instrumental magnitudes into apparent magnitudes with the standard relation: $m a g_{\mathrm{app}}=m a g_{\mathrm{inst}}-Z_{\mathrm{p}}-e x t * A M$ where $m a g_{\text {app }}$ and $m a g_{\text {inst }}$ are respectively the apparent and instrumental magnitudes, $Z_{\mathrm{p}}$ the zero-point, ext the extinction and $A M$ the airmass. The observations were performed through an $A M$ close to 1.

We also carried out optical spectroscopy with EMMI. We took 12 spectra with the grisms \#1 covering 4000 to $10000 \AA$ with a resolution between 200 and $500 \AA$ (depending on the wavelength). Each spectrum had an exposure time of $300 \mathrm{~s}$, giving a total integration time of $60 \mathrm{~min}$. In order to extract spectra and perform wavelength and flux calibrations, we used the IRAF noao.twodspec package.

\subsection{Radio data analysis}

We observed SWIFT J1753.5-0127 with the NRAO10 Very Large Array (VLA) at 1.4, 4.9, 8.5 and $15 \mathrm{GHz}$ (respectively 21, 6, 3.5 and $2.0 \mathrm{~cm}$ wavelength) on $2005 \mathrm{Au}-$ gust 11 from 4:20 to 8:50 U.T. (average MJD 53593.28) with the VLA in its $\mathrm{C}$ configuration. The receiver setup included two IF pairs of $50 \mathrm{MHz}$ bandwidth each. We obtained 20 min snapshots at 1.4, 4.9 and $15 \mathrm{GHz}$. We also derive a $\sim 2.5$ hour lightcurve at $8.5 \mathrm{GHz}$. The observations were conducted as follows: $10 \mathrm{~min}$ scans on SWIFT J1753.5-0127 preceded and followed by $1 \mathrm{~min}$ scans on the VLA phase calibrator J1743-038 (located $3.4^{\circ}$ apart). The primary flux density calibrator used was J0137+331 (3C 48). The data were reduced using standard procedures within the NRAO aips software package.

\section{RESULTS OF THE ANALYSIS}

\subsection{X-ray position}

In the combined IBIS/ISGRI images obtained during the hard outburst, the transient source SWIFT J1753.5-0127 was detected at 440, 320, 174 and $22 \sigma$ respectively in the $20-40,40-80,80-160$ and $160-$ $320 \mathrm{keV}$ energy bands. Figure 2 shows the $20-40 \mathrm{keV}$ IBIS/ISGRI mosaic: three other sources were detected, the neutron star $4 \mathrm{U} 1812-12(34 \sigma)$, the X-ray burster Ser X-1 $(14 \sigma)$ and the INTEGRAL obscured source named IGR J17303-0601 $(12 \sigma)$. The best-fit position found by IBIS/ISGRI over $\sim 176$ ks exposure in the 20 $40 \mathrm{keV}$ mosaic sky image was $\alpha_{\mathrm{J} 2000}=17^{\mathrm{h}} 53^{\mathrm{m}} 28^{\mathrm{s}} .4$ and $\delta_{\mathrm{J} 2000}=-01^{\circ} 27^{\prime} 17^{\prime \prime}$, with an accuracy of $14^{\prime \prime}$ at the $90 \%$ confidence level (Gros et al. 2003). This position is compatible with the Swift/XRT location (Burrows et al. 2005) as the angular separation between ISGRI and $\mathrm{XRT}$ is $7^{\prime \prime}$ only. It is also consistent with the most precise position of SWIFT J1753.5-0127 derived from the Swift/UVOT data (Still et al. 2005) since the offset is only $11^{\prime \prime}$ (within the error circle). The high-energy source and

\footnotetext{
${ }^{10}$ The National Radio Astronomy Observatory is a facility of the National Science Foundation operated under cooperative agreement by Associated Universities, Inc.
} 
the UV/optical/radio counterparts are therefore unambiguously associated to the X-ray transient source.

\section{2. $X$ and $\gamma$-ray light curves}

Figure 1 shows the $1.2-12 \mathrm{keV} R X T E /$ ASM daily average $\mathrm{XN}$ light curve from just before its discovery, during its outburst rise and peak (in 2005 July) and down to its return to a lower ASM level (almost undetectable around 2006 mid-June). From the end of 2005 May up to July 9, the ASM average count rate (where $1 \mathrm{Crab}=75 \mathrm{cts} \mathrm{s}^{-1}$ ) increased and its flux reached the maximum value of $\sim$ $200 \mathrm{mCrab}$ (MJD 53560). The flux then decreased to $14 \mathrm{mCrab}$ (2005 October 10, MJD 53650) before the source became nearly undetectable by the ASM. Assuming an exponential shape for both the rise and the decay phases seen in the ASM, we obtained time constants of $4.5 \pm 0.2$ (rise) and $32.0 \pm 0.2$ (decay) days respectively. The characteristic decay time we derived is compatible with the usual behavior of XN in outburst (Tanaka \& Shibazaki, 1996; Chen et al. 1997) like, e.g., XTE J1720-318 (Cadolle Bel et al. 2004).

The large arrow overplotted on Figure 1 indicates the interval covered by the dedicated INTEGRAL ToO observations discussed in this work, for which we also triggered simultaneous optical, NIR and radio observations. Between MJD 53592-53594.4, the IBIS/ISGRI count rate was almost constant at $43 \mathrm{cts} \mathrm{s}^{-1}$ (Figure 3 top) which corresponds to a significative flux of $\sim 205$ mCrab between 20-320 keV. Figure 3 (bottom) also shows that the HR between the $20-40$ and $40-80 \mathrm{keV}$ energy bands was compatible with being constant $(\sim 0.75)$. The source was observed in the decay phase of its outburst which started (as seen in Figure 1) around MJD 53560. However, the source was still bright in the INTEGRAL/IBIS energy band at the time of our observations. This favors the scenario that the source probably stayed in the LHS during (see also Ramadevi \& Seetha 2006) and after its outburst (see Miller et al. 2006). In particular, SWIFT J1753.5-0127 did not show transitions to a softer state: we will discuss this issue in Section 3.4.

\subsection{Timing variability from high-energy data}

We produced a Power Density Spectrum (PDS) from the PCA high time resolution light curves with POWSPEC V1.0. The PDS was generated on several intervals of $256 \mathrm{~s}$ between $3.9 \times 10^{-3}$ and $1024 \mathrm{~Hz}$. The results were averaged together and the resulting PDS is shown in Figure 4. It was corrected for white noise before fitting. Since above $20 \mathrm{~Hz}$ the PDS is compatible with Poisson noise, we restricted our fitting to the $3.9 \times 10^{-3}-20 \mathrm{~Hz}$ frequency range. The continuum is well represented by the sum of 2 broad zero centered Lorentzians while an additional third one is needed to account for a QPO around $0.24 \mathrm{~Hz}$. Even if it is faint ( $5.4 \%$ r.m.s.), an F-test confirms that this component is required $(99.95 \%$ level probability). Note that in the case of a broad Lorentzian, the frequency at which the feature attains its maximum (in a $\nu P_{\nu}$ representation) is defined by $\nu_{\max }=\sqrt{\nu_{0}^{2}+\Delta^{2}}$ (Belloni et al. 2002) where $\Delta$ is the half width at half maximum. Hence in our case this leads to $\nu_{\max }=\Delta$. This model of 3 Lorentzians provides a relatively good description of the PDS with a $\chi^{2}=197.1$ for 163 degrees of freedom (dof). The normalized PDS (after Leahy et al. 1983) and the individual components are represented in Figure 4 The parameters of each Lorentzian are reported in Table 2, The QPO mean value is lower than the $0.6 \mathrm{~Hz}$ QPO reported after the peak of the outburst (Morgan et al. 2005; Ramadevi \& Seetha 2006). This trend (decrease of the QPO frequency during the decrease of the eruption) is sometimes observed in other BHCs and has been associated to the increase of the inner radius of the accretion disk (Kalemci et al. 2001, 2002; Rodriguez et al. 2002, 2004a; Belloni et al. 2005). However, we can not exclude another interpretation for the QPO based on the pulsation modes of the corona (Shaposhnikov \& Titarchuk 2006). We did not detect the QPO in either JEM-X nor ISGRI data: this is probably due to their lower collecting area than PCA and to the faintness of the QPO at the time of our INTEGRAL ToO. In any case the high level of band-limited noise $(\sim 27 \%$ r.m.s., see Table 2) we observed is typical of the LHS of $\mathrm{XN}$.

\section{4. $X$ and $\gamma$-ray spectral results}

Figure 3 shows the flux and HR measured during the INTEGRAL high-energy observations. The IBIS/ISGRI flux appears to be constant as well as the HR $(\sim 0.75)$ during the course of our ToO observations. As there is no significant variation in the HR, we therefore used the whole data from JEM-X, IBIS/ISGRI and SPI of this hard outburst to build up an average spectrum on a wide band together with the simultaneous PCA and HEXTE data obtained during our INTEGRAL ToO. We added $3 \%$ systematic errors for JEM-X (in the $5-25 \mathrm{keV}$ range) and SPI (in the $22-600 \mathrm{keV}$ range) and $2 \%$ for IBIS (in the $18-320 \mathrm{keV}$ range). Using XSPEC v11.3.0 (Arnaud et al. 96), we fitted the resultant spectra simultaneously with PCA (3-25 keV) and HEXTE (20-100 keV). In order to account for uncertainties in the cross-calibration of each instrument, a multiplicative constant was added in the spectral fits to each instrument data set: it was frozen to 1 for PCA and set free for JEM-X, IBIS/ISGRI, SPI and HEXTE.

Following the approach described in Cadolle Bel et al. (2006) when modelling the LHS spectra of Cygnus X1 , we fitted our simultaneous $\mathrm{X} / \gamma$-ray data from the $R X T E$ and INTEGRAL satellites to different spectral distribution functions. We added progressively each spectral component to account for possible presences of Comptonization (Titarchuk 1994), multicolor black body disk (Mitsuda et al. 1984), reflection or Fe line. We then tested if each component was required by the improvement in $\chi_{\text {red }}^{2}$. We always used a fixed absorption column density $N_{\mathrm{H}}$ of $2 \times 10^{21}$ atoms $\mathrm{cm}^{-2}$, taking the value obtained with our optical data (see Section 3.5). Indeed, we can not constrain it with the PCA nor the JEM-X data. First, we tried to fit simultaneously all the data with the Comptonization model of Titarchuk (1994) convolved by absorption ( CONS $^{*}$ WABS $^{*}$ COMPTT in XSPEC notation). We obtained a seed photon temperature $k T_{0}$ of $0.51 \pm 0.08 \mathrm{keV}$, a plasma temperature $k T_{\mathrm{e}}$ of $88 \pm 14 \mathrm{keV}$ and a plasma 
optical depth $\tau$ of $0.67 \pm 0.14$. However, this model gave an unacceptable fit $\left(\chi_{\text {red }}^{2}=2.14\right.$ with 322 dof $)$ to the data. Including a multicolor black body disk did not improve the fit, with most of the large residuals showing up below $20 \mathrm{keV}$ between the PCA and JEM-X data sets and in the HEXTE data between $20-30 \mathrm{keV}$ and above $70 \mathrm{keV}$. The reflection (Magdziarz \& Zdziarski 1995) is usually employed to account for a soft excess around $10 \mathrm{keV}$. This latter component (noted REFLECT in XSPEC) models the $\mathrm{X}$-ray reflection of the comptonized radiation from neutral or partially ionized matter, presumably the optically thick accretion disk (see also Done et al. 1992; Gierliński et al. 1997, 1999). Adding this component with an inclination angle equal to $63^{\circ}$ (default), we obtained a significant decrease in $\chi_{\text {red }}^{2}(1.60)$ but a very high plasma temperature. This model gave an improved fit but, again, it was still unacceptable.

As the JEM-X and HEXTE instruments overlap the energy bands of, respectively, PCA and IBIS/ISGRI (and SPI) and as these latter instruments are supposed to be the best calibrated, we tried to fit simultaneously only the data from PCA, IBIS/ISGRI and SPI. With the CONS*WABS*(REFLECT ${ }^{*}$ COMPTT) model, we obtained a reasonable $\chi_{\text {red }}^{2}$ of 1.17 (with $121 \mathrm{dof}$ ). Our best-fit model has the following parameters: a seed photon temperature $k T_{0}$ of $0.54_{-0.07}^{+0.04} \mathrm{keV}$, an electron temperature $k T_{\mathrm{e}}$ of $150 \pm 26 \mathrm{keV}$, an optical depth $\tau$ of $1.06 \pm 0.02$ and a reflection fraction $\Omega / 2 \pi=0.32 \pm 0.03$. Normalization constants between IBIS/ISGRI and SPI were respectively equal to 0.97 and 1.20 when PCA was frozen to 1 . Note that these parameters (even if the plasma temperature is higher when the reflection component is added) are compatible with the ones obtained previously while fitting simultaneously all the data (from PCA, JEM-X, HEXTE, IBIS/ISGRI and SPI). This best-fit model over-plotted on the data from PCA, IBIS/ISGRI and SPI is reported in count units in Figure 5. We have rebinned the IBIS/ISGRI (above $\sim 300 \mathrm{keV}$ ) and SPI (above $\sim 500 \mathrm{keV}$ ) data at the level of $3 \sigma$. Figure 6 also shows our best-fit model with its components overplotted on the same data in $E F(E)$ units $\left(\mathrm{keV} \mathrm{cm}^{-2} \mathrm{~s}^{-1}\right)$.

The parameters we derive are compatible with the source being in the LHS. The relatively high plasma temperature indicates that the Comptonizing medium (corona) remains hot: this is probably due to the weak disk emission unable to efficiently cool down the corona by Compton scattering. The derived value $(\sim 0.33)$ of the Comptonization parameter $y$, given by $k T_{\mathrm{e}} / m_{\mathrm{e}} c^{2} \operatorname{Max}(\tau$, $\left.\tau^{2}\right)$, is typical of a LHS, as fully discussed in Cadolle Bel et al. (2006) for Cygnus X-1. We therefore conclude that the source was very hard during the eruptive phase with a behavior and spectral Comptonization parameters typical of a $\mathrm{BH}$ in LHS.

\subsection{Optical and NIR results}

Concerning the photometry obtained on 2005 August 11 (U.T. 02) with EMMI, the apparent magnitudes we derived are: $B=16.73 \pm 0.02, V=16.46 \pm 0.02$, $R=16.15 \pm 0.02$ and $I=15.64 \pm 0.03$. They are nearly 0.5 mag fainter than the apparent magnitudes obtained by Still et al. (2005) and Torres et al. (2005a, b) during the period of July 1-11, probably because our observations were obtained later in the outburst. Torres and co-workers suggested a slow change rate at a level of 0.1 mag per week. This is exactly the behavior we have observed later and it is compatible with the evolution of an XN in outburst. The same trend was observed during subsequent monitoring with REM: indeed, the flux in the $R$ filter results to be almost constant at the average level of $\sim 16.45 \mathrm{mag}$ on August 17 and of $\sim 16.60$ on August 27. REMIR photometry reveals a NIR counterpart with $J=15.35 \pm 0.06$, $H=15.11 \pm 0.07$ and $K=14.73 \pm 0.15$ mag on August 11.

Figure 7 represents the derived EMMI spectrum in erg $\mathrm{cm}^{-2} \mathrm{~s}^{-1} \AA^{-1}$ units and calibrated with the EG274 standard spectrophotometric star: the continuum is blue, suggesting the emission from an accretion disk, and it shows many telluric lines at respectively 6300, 6900, 7200, 7700 and $8300 \AA$. Interstellar lines at 5800, 5900 (NaI Doublet) and $6300 \AA$ are present. We do not observe any clear absorption nor emission lines apart from the $\mathrm{H} \alpha$ (around $6500 \AA$ ) and the He I (around 5800, 6600 and $7200 \AA$ ) lines: they are visible in absorption (but very faint). They probably emanate from the accretion disk as suggested by the blue continuum. The non-detection of any emission line differs from the results of observations reported earlier during the outburst on July 3 (Torres et al. 2005a): the spectra showed then a blue continuum with $\mathrm{H} \alpha$ being the only emission line detected in the spectrum with an Equivalent Width $(\mathrm{EW})$ of $\sim 3 \AA$ and a Full Width at Half Maximum (FWHM) of $2000 \mathrm{~km} \mathrm{~s}^{-1}$. The NaI doublet at 5890/96 $\AA$ was also evident with $\mathrm{EW} \sim 1.5 \AA$. One week after (Torres et al. 2005b), a broad double-peaked $\mathrm{H} \alpha$ emission was detected each night (July 7, 10, 11 and 12) with mean EW of $3 \AA$, mean double-peacked separation of $1200 \mathrm{~km} \mathrm{~s}^{-1}$ and a FWHM around $2000 \mathrm{~km} \mathrm{~s}^{-1}$. This is characteristic of the outer accretion disk in X-ray binaries with a low mass companion star. He II (4686 $\AA$ ) emission was highly variable both in terms of strength and profile shape, ranging between $\sim 3$ and $\sim 5 \AA$ in EW. On August 11, the $\mathrm{H} \alpha$ line has almost disappeared and is not anymore double-peaked: the evolution of this line is typical for LMXB (novae) in outburst. The low amplitude and EW of the lines suggest that the disk has a fainter contribution at the time of our simultaneous optical/INTEGRAL observations, as also confirmed by the fact that the $B-V$ value stayed almost constant (at 0.3 mag) during and after the outburst. Comparisons of the spectra obtained between July and mid-August show the expected behavior of LMXBs in eruption: bright contribution of a disk in optical, followed by a decrease of this contribution simultaneously to a decrease of the soft X-ray flux.

Finally, we could determine the column density along the line of sight, using $\mathrm{EW}$ of the NaI Doublet $(1.36 \pm 0.15 \AA)$. According to Munari \& Zwitter (1997), $E_{\mathrm{B}-\mathrm{V}}=0.25 \times \mathrm{EW}_{\mathrm{NaI}}$ : therefore we obtain $E_{\mathrm{B}-\mathrm{V}}=$ $0.34 \pm 0.04 \mathrm{mag}$. Using the formula of Bohlin et al. (1978) $N\left(H_{I}+H_{2}\right)=5.8 \times 10^{21} \times E_{\mathrm{B}-\mathrm{V}}$, we calculate $N_{\mathrm{H}}$ and we get $1.97 \pm 0.23 \times 10^{21}$ atoms $\mathrm{cm}^{-2}$. This value is well 
Cadolle Bel et al.

consistent with the absorption determined by Swift/XRT (Morris et al. 2005). Depending on which total galactic absorption we get $\left(\sim 1.7 \times 10^{21}\right.$ atoms $\mathrm{cm}^{-2}$ from Dickey \& Lockman 1990 commonly used by the X-ray community or $\sim 2.7 \times 10^{21}$ atoms $\mathrm{cm}^{-2}$ with Schlegel et al. 1998), the source $N_{\mathrm{H}}$ is below or comparable within errors with the total galactic column density in this direction so the source can not be located at more than $10 \mathrm{kpc}$. Besides, it is situated at least at more than $2-3 \mathrm{kpc}$ in order to be compatible with its absorption and its high latitude value $\left(l=12.9^{\circ}\right)$. Therefore, a probable distance for the source is between 4 and $8 \mathrm{kpc}$ : we took the average value of $6 \mathrm{kpc}$ in the following.

\subsection{Radio results}

We produced images using a natural weighting scheme. Considering the angular resolution of the VLA (the synthesized beam), we detected at all observed frequencies a point-like radio counterpart (angular radius $<4^{\prime \prime}$ ) at a position compatible with the MERLIN one. The obtained flux density at each frequency $\left(S_{\nu}\right)$ and the fitted spectral index $\alpha$ (where $S_{\nu} \propto \nu^{+\alpha}$ ) in different frequency ranges are quoted in Table 3 All errors are at the $1 \sigma$ level and we show in parentheses the expected r.m.s. sensitivities for the corresponding amounts of observing time. The discrepancy between the expected (0.04) and observed (0.15) error values at $1.4 \mathrm{GHz}$ is because we could only image the source properly after excluding the galactic diffuse emission detected on short baselines (hence increasing the noise). The discrepancy at $4.9 \mathrm{GHz}$ is due to the fact that we considered the higher error given by the jmfit procedure (Gaussian fitting). The source was slightly variable during our long $8.5 \mathrm{GHz}$ observing run (see below), and different flux densities were obtained depending on the measuring method: flux density within a box (imstat), peak and integral flux of a Gaussian fit (jmfit). Therefore we give the average value of $0.7 \pm 0.1$. Finally, the VLA data at $15 \mathrm{GHz}$ could only provide a $3 \sigma$ upper limit of $0.60 \mathrm{mJy}$, which will not be considered hereafter in our analysis and interpretation.

The flux densities as a function of observing frequency are shown in logarithmic scale in Figure 8 . A flat -or slightly inverted- spectrum with $\alpha=+0.03 \pm 0.03$ is found after excluding the $15 \mathrm{GHz}$ upper limit point. We also show in Figure 9 the radio lightcurve obtained at $8.5 \mathrm{GHz}$ in MJD units. The gap corresponds to the observing time at the other frequencies. Each data point has been obtained after measuring the flux density in an image produced with the corresponding $10 \mathrm{~min}$ snapshot. The final two data points were obtained after observations conducted at low elevations $\left(18\right.$ and $\left.15^{\circ}\right)$. Although the gain corrections due to elevation have been taken into account, the flux density of the phase calibrator also decreases sligthly in this last part of the observation, while the rms of its phases increases significantly. Therefore, since the target flux density was too low to self-calibrate the phases, we consider that the measured flux densities and corresponding uncertainties of these two data points could be both underestimated. The average value of all the data points is $0.60 \pm 0.11 \mathrm{mJy}(0.61 \pm 0.09 \mathrm{mJy}$ if we exclude the last two points). This value is lower but compatible with the one obtained above after performing a single image with all the data. This difference can be due to the sligthly variable behavior seen in Figure 9, Indeed, even if excluding the last two data points, a $\chi^{2}$ test reveals that the data are not compatible with a constant value at the $99.5 \%$ confidence level : this confirms that the radio flux was variable at the level of $\sim 30 \%$.

\section{DISCUSSION}

\subsection{Spectral energy distribution}

We calculated for radio, NIR, optical and X-ray data the corresponding flux value in $\nu F_{\nu}$ units $\left(\mathrm{erg} \mathrm{cm}^{-2} \mathrm{~s}^{-1}\right)$ corrected for extinction. Using our estimated value of $E_{\mathrm{B}-\mathrm{V}}$ equals to $0.34 \pm 0.04 \mathrm{mag}$ and the formula of Predehl \& Schmitt (1995), we found $A_{V} \sim 3.1 \times E_{\mathrm{B}-\mathrm{V}}=$ $1.05 \pm 0.12$ mag. We then obtained $A_{\lambda}$, respectively in the $B, R$ and $I$ filters (i.e. $\lambda$ equals 4400,7100 and $9700 \AA$ ) and in the REM filters with the formula of Mathis et al. (1992) and Cardelli et al. (1989) depending on the wavelength. We got $A_{B}=1.54 \pm 0.18$, $A_{R}=0.68 \pm 0.08, A_{I}=0.40 \pm 0.05, A_{J}=0.30 \pm 0.12$, $A_{H}=0.20 \pm 0.12$ and $A_{K}=0.12 \pm 0.12 \mathrm{mag}$. Then, the magnitudes were dereddened and we finally estimated the corresponding fluxes and errors in units of erg cm- $\mathrm{cm}^{-2} \mathrm{~s}^{-1}$. RXTE and INTEGRAL data were added to this plot which represents the Spectral Energy Distribution (SED) of SWIFT J1753.5-0127. We show our results from radio to $\mathrm{X} / \gamma$-rays in Figure 10 using a logarithmic scale: the $\mathrm{X}$-axis is labeled as $\log (\nu)$ units and Y-axis in $\log \left(\nu \mathrm{F}_{\nu}\right)$.

From the analysis of the SED, we can confirm that the source shows all the usual signs of a $\mathrm{BH} \mathrm{XN}$ in the LHS. Firstly, we immediately note that the source has a hard power law index in the high-energy domain. It is also clear that a simple power law can not fit all the data together from radio to $\mathrm{X} / \gamma$-rays; at least two breaks are necessary in the SED. Secondly, the radio emission is usually interpreted in this state as synchrotron emission emanating from a self-absorbed compact jet (Blandford \& Konigl 1979). So in our case the flat radio power law component could be explained by the optically thick synchrotron emission from the jet (Markoff et al. 2005). From the SED we derive the power law index $\alpha$ (in $S_{\nu}$ ): $\sim 0.03$ in the radio (see 3.4) and $\sim 1.1$ in the optical/NIR. This latter value is compatible with the disk emission according to the results reported by Hynes et al. (2005) on several $\mathrm{X}$-ray binaries and with the value observed (for example) in XTE J1118+480 by Chaty et al. (2003). If we extend the power law spectrum derived from the radio data points up to the NIR-optical frequencies with the same slope, the extrapolated flux is compatible with the first NIR points, in the $K$ band (see Figure 10), but not with the rest of the measures. This suggests that the synchrotron emission from the compact jet of SWIFT J1753.5-0127 may contribute significantly to the $K$ band (as seen in certain $\mathrm{BH}$ in LHS, e.g., Russell et al. 2006 and references therein) but little to the entire NIR-optical flux. This latter is probably dominated by the disk emission.

The SED shown in Figure 10 reveals that at least three distinct contributions are necessary to account for our multiwavelength data (as a single power law can not do 
so): the jet, the disk and the corona. The necessity of an extra component to the jet to take into account our $B, V, R, I, J, H$ and $K$ measured fluxes may represent part of the black body disk contribution (thermal emission) instead of the (faint) companion. However, we do not have enough data in the required energy range $(0.1-2 \mathrm{keV})$ to constrain and modelize the SED: the disk contribution is faint in our data range. We can only state that a break is necessary in the previous power law (from radio) and that at least another component than the jet contributes in the NIR-optical range. Then another break between the NIR-optical and soft X-ray data $(<1 \mathrm{keV})$ is needed (in addition to the high-energy break around 50-100 keV of the X/gamma-ray LHS spectrum) to model our X-ray $/ \gamma$-ray data points of the source from $R X T E$ and INTEGRAL: these points have indeed a steeper slope than the radio, IR and optical ones. The shape of the SED is similar to the ones observed for transient LMXBs (e.g., XTE J1118+480, Chaty et al. 2003; XTE J1720-318, Chaty \& Bessolaz 2006).

\section{2. $X$ and $\gamma$-ray constraints}

During the broad-band ( $3 \mathrm{keV}-1 \mathrm{MeV})$ observations of SWIFT J1753.5-0127 presented in this paper, the source was detected in a LHS well characterized by a thermal Comptonization model modified by reflection. The best-fit parameters we obtain through the $R X T E$ and INTEGRAL data are consistent with those found in $\mathrm{BH}$ binaries in the LHS (McClintock \& Remillard 2006). The Comptonization results reported in this work are typical of a LHS as seen in the prototypical BH Cygnus X-1 (Cadolle Bel et al. 2006). Indeed, our reported results obtained on Cygnus X-1 during the LHS indicated a high Comptonization parameter $(\sim 0.51)$ like in the present case $(\sim 0.33)$. The reflection fraction we derived is also compatible with values previously found for sources in the LHS. The high peak luminosity, the fast rise, the slow decay time scales and the bright hard state with spectral parameters and variability properties typically observed in other (dynamically confirmed) BH transients in LHS like e.g. XTE J1550-564 (Sobczak et al. 2000; Rodriguez et al. 2003), GRO J1655-40 (Sobczak et al. 1999, see also McClintock \& Remillard 2006) or Nova Persei 92 (Roques et al. 1994, Denis et al. 1994, Finoguenov et al. 1996) clearly show that SWIFT J1753.5-0127 is very likely a new XN and BHC.

The fact that we could not constrain the disk emission in LHS may be due to its very low inner temperature which implies a negligible contribution at more than $3 \mathrm{keV}$. This is compatible with the results obtained seven months later by Miller et al. (2006) who reported that the disk component, well constrained by the $X M M /$ EPIC-pn spectrum, was very cold $(0.22 \mathrm{keV})$. Besides, the lack of strong Fe line is surprising with our reflection fraction value but a possible interpretation is that the $\mathrm{Fe}$ line is originated from the outer disk and we do not observe it due to inclination effect of the orbit.

While our data start at $3 \mathrm{keV}$, leading to a possible underestimation of the bolometric luminosity, we derive an unabsorbed $2-11 \mathrm{keV}$ flux of $1.5 \times 10^{-9} \mathrm{erg} \mathrm{cm}^{-2} \mathrm{~s}^{-1}$, an unabsorbed 20-500 keV flux of $8.3 \times 10^{-9} \mathrm{erg} \mathrm{cm}^{-2} \mathrm{~s}^{-1}$ and a bolometric flux (extrapolated from $0.01 \mathrm{keV}$ to $10 \mathrm{MeV}$ ) of $1.3 \times 10^{-8}$ erg $\mathrm{cm}^{-2} \mathrm{~s}^{-1}$. This corresponds to an unabsorbed bolometric luminosity of $5.77(d / 6 \mathrm{kpc})^{2} \times 10^{37} \mathrm{ergs} \mathrm{s}^{-1}$, well below the Eddington regime for a stellar mass BH. Miller et al. (2006) reported more than 7 months after our observations that the unabsorbed flux in the $0.5-10 \mathrm{keV}$ range was $3.9 \times 10^{-10}$ erg $\mathrm{cm}^{-2} \mathrm{~s}^{-1}$ while during our observations we get the higher value of $2.1 \times 10^{-9} \mathrm{erg} \mathrm{cm}^{-2} \mathrm{~s}^{-1}$. Stellar mass $\mathrm{BH}$ accreting at or below $10^{-2} L_{\mathrm{Edd}}$ are found in the LHS (McClintock \& Remillard 2006). These results are again compatible with the fact that the source was in the LHS during the rise and the decline of the outburst.

On the other hand, we can take the bolometric flux between $0.01 \mathrm{keV}$ and $10 \mathrm{MeV}$, compute the bolometric luminosity for different distances to SWIFT J1753.5-0127 and derive the minimum compact object mass to guarantee that this corresponds to less than $5 \%$ of the Eddington luminosity, as seen in BH in the LHS (Maccarone et al. 2003). These results are shown in the first two panels of Figure 11 and in Table 4 (for the minimum compact object mass). If the overall $0.01 \mathrm{keV}$ to $10 \mathrm{MeV}$ spectrum had the same shape during the maximum of the X-ray outburst, since the RXTE/ASM X-ray flux was a factor of 3 higher than during our observations, the derived minimum compact object masses should be a factor of 3 higher than those quoted in Table 4 . However, since we do not have precise broad-band spectral informations during the maximum of the outburst, we will use hereafter the bolometric X-ray flux at the time of our observations, as well as the derived minimum compact object masses for this flux.

Besides, the QPO frequency evolves from the beginning of the outburst to our INTEGRAL observations: it decreases from the average value of 0.60 (Morgan et al. 2005) to $0.24 \mathrm{~Hz}$. If the low frequency QPO is related to the inner disk radius, this result would indicate that this radius increases during the decay of the outburst as seen before in, e.g., XTE J1550-564 (Rodriguez et al. 2004a). This tendency is consistent with the late stages of a LMXB outburst. These results are also compatible with the ones reported by Miller et al. (2006) who found, seven months after our ToO, a strong, band-limited variability noise (30\% r.m.s. noise amplitude in the different range $0.01-$ $100 \mathrm{~Hz}$ ) which is typical of the LHS in accreting BH, while they do not detect any QPOs anymore. On the other hand, the QPO frequency evolution may have another possible interpretation: it could be linked to pulsational modes in the corona as recently suggested by Shaposhnikov \& Titarchuk (2006).

\subsection{Optical constraints}

The blue optical spectrum seems to be the emission from an accretion disk while the absence of strong emission lines points towards a late-type companion star. If this is the case we should expect a fainter quiescent magnitude (consequently much higher than the $V=15.9 \pm 0.1 \mathrm{mag}$ value 
while in outburst reported by Still et al. 2005). Usually, the optical magnitudes of BH XN change of about 5 mag from the peak of the outburst to the quiescence level as observed in other eruptions (e.g., GS 1354-64, Brocksopp et al. 2001). This trend is similar to the one shown by our results in spite of the fact that SWIFT J1753.5-0127 magnitudes increase more slowly. Besides, the source is not visible in archival images. Comparing with nearby faint USNO-B1.0 stars (Monet et al. 2003), we estimate a fainter quiescent visual magnitude (i.e. above $19.5 \mathrm{mag}$ from $R>19.0 \pm 0.5$ and $B>20.0 \pm 0.5)$. Using for example the absolute visual magnitudes from Ruelas-Mayorga (1991), even for the less luminous intermediate type giant companions in the range F8-G2 III, the distance to the source should be $\sim 15 \mathrm{kpc}$, implying a very high minimum $\mathrm{BH}$ mass of $\sim 55 M_{\odot}$ to guarantee $L_{\mathrm{bol}}<5 \% L_{\mathrm{Edd}}$. The situation would be much worse for other spectral types. Clearly, an intrinsically fainter donor is required. To have a $\mathrm{BH}$ mass below $\sim 10 M_{\odot}$ (for $L_{\text {bol }}<5 \% L_{\mathrm{Edd}}$ ), the distance has to be below $6 \mathrm{kpc}$, requiring for the companion star a spectral type later than G0 V, for a $V=19.5$ quiescent magnitude, or later than $\mathrm{K} 0 \mathrm{~V}$, if the quiescent magnitude is $V=21.0$ (i.e., $\sim 5$ magnitudes fainter than the $V=15.9 \pm 0.1 \mathrm{mag}$ value while in outburst). Therefore, the optical results suggest a (main sequence) type $\mathrm{K}$ or $\mathrm{M}$ companion rather than earliest types, ranging SWIFT J1753.5-0127 in the LMXB class. However, as the emission is dominated by the disk and we could not detect any clear absorption lines, it is impossible to derive for sure the spectral type of the companion. In addition, there is no change in the $E_{\mathrm{B}-\mathrm{V}}$ value.

The value of hydrogen column density we have derived thanks to the EW of the NaI Doublet, $N_{\mathrm{H}}=(1.97 \pm$ $0.23) \times 10^{21}$ atoms $\mathrm{cm}^{-2}$, is very similar to the one measured with by Swift/XRT, of $N_{\mathrm{H}} \sim 2.0 \times 10^{21}$ atoms cm $^{-2}$ (Morris et al. 2005). There is no need to advocate for extra intrinsic absorption around the X-ray emitting source and a reasonable distance for this LMXB, probably located in the Galactic Halo, could be $6 \mathrm{kpc}$. This corresponds to a galactic height of $1.3 \mathrm{kpc}$ (see lower panel of Figure 11). This has to be compared with the LMXB Galactic Halo sources XTE J1118+480, located at $1.72 \pm 0.10 \mathrm{kpc}$ (Gelino et al. 2006) with a height above the Galactic Plane of $1.5 \pm 0.1 \mathrm{kpc}$, and Scorpius X-1, located at $2.8 \pm 0.3 \mathrm{kpc}$ (Bradshaw et al. 1999) with a height of $1.1 \pm 0.1 \mathrm{kpc}$. Therefore, a distance of $6 \mathrm{kpc}$ to SWIFT J1753.5-0127 would place it at a similar height above the Galactic Plane than those of XTE J1118+480 and Scorpius X-1.

\subsection{Radio constraints and discrepancy with the radio/X-ray correlation}

The point-like nature of the source during the MERLIN observations provides a brightness temperature of $T_{\mathrm{b}}=$ $1.0 \times 10^{4} \mathrm{~K}$ (see, e.g., Martí et al. 1998), thus practically ruling out thermal emission mechanisms. On the other hand, the flat radio spectrum of SWIFT J1753.5-0127 is similar to the ones typically found in $\mathrm{BH}$ during LHS (e.g., Fender et al. 2006 and references therein). This is compatible with (and usually interpreted as) synchrotron radiation produced in a partially self-absorbed conical and compact jet (Gallo et al. 2003, Fender et al. 2004, Corbel et al. 2004). It is not resolved in our VLA data because we do not have enough angular resolution, contrary to the cases of Cygnus X-1 or GRS 1915+105 (Stirling et al. 2001; Fuchs et al. 2003; Ribó 2005) observed with the VLBA. Even MERLIN could not resolve the source.

Corbel et al. (2003) then Gallo et al. (2003) found the following correlation between the X-ray flux and the radio flux density for $\mathrm{BH}$ in the LHS: $F_{\text {rad }}=k F_{\mathrm{X}}^{+0.7}$ (with $k=223 \pm 156$ for all fluxes scaled to a distance of $1 \mathrm{kpc}$ ). We have used our measured unabsorbed X-ray flux $\left(F_{2-11 \mathrm{keV}}=1.5 \times 10^{-9} \mathrm{erg} \mathrm{cm}^{-2} \mathrm{~s}^{-1}\right)$ to compute the expected radio flux density according to their correlation by using different possible distances to SWIFT J1753.5-0127. We proceed as follows: for each considered distance we scaled our measured X-ray flux to the one we should detect at $1 \mathrm{kpc}$ distance, we then computed the expected radio flux at $1 \mathrm{kpc}$ according to the correlation and we finally derived the expected radio flux we should detect if the source is placed at the considered distance. The obtained results are quoted in the third column of Table 4 and are shown in the third panel of Figure 11. It is clear from this Figure that the measured value is one order of magnitude lower than the expected one according to the radio/X-ray correlation, even for the highest possible distances to the source. We have performed the same analysis using the recent results of Gallo et al. (2006) who found a lower index of $\sim 0.6$ in the radio/X-ray correlation: the same procedure, using this slightly flatter correlation (which only modifies significantly the lower X-ray flux part), provides very similar results. Indeed, since our source is approximately at the level of $0.1 \mathrm{Crab}$ (for a distance of 1 $\mathrm{kpc}$ ) and at the level of $2 \mathrm{Crab}$ (for a distance of $5 \mathrm{kpc}$ ), we are in the X-ray luminous part of the original Corbel et al. (2003) and Gallo et al. (2003) correlations: the new flatter correlation does not change significantly our results and, in any case, they are well within the errors of those obtained with the original correlation in this part of the diagram.

For comparison, the MERLIN observation performed on MJD 53555 (centered on 2005 July 3 at 23:00) took place when the $R X T E /$ ASM X-ray flux was higher with a factor of $2.9\left(13 \pm 1\right.$ compared to $4.5 \pm 0.5$ count s $\left.^{-1}\right)$. From this we can predict a factor of 2.15 higher for the radio emisssion while the radio flux density was 3 times higher. Therefore, similar (although slightly smaller) discrepancies are obtained in this case. Therefore, this would point to a different track in the radio/X-ray fluxes correlation diagram than the one previously observed (Gallo et al. 2003). This could indicate that the compact jet formation and luminosity may be able to evolve differently than previously thought (see also Corbel et al. 2004 for XTE J1650-500; Rodriguez et al. 2006b for IGR J17497-2821 and Chaty 2006 for XTE J1720-318).

\section{CONCLUSIONS}

In recent years, it has become apparent that in the LHS, the BH binaries become bright in radio (Fender et al. 2006 for a complete review) and display clear 
correlations between the X-ray and radio luminosities (Corbel et al. 2003; Gallo et al. 2003; Gallo et al. 2006; Russell et al. 2006) as observed, for example, in the confirmed BH system Cygnus X-1 (Brocksopp et al. 1999; Gleissner et al. 2004; Nowak 2005). Models where the base of a compact jet plays a major role in the physical processes of such BH systems have been proposed (Markoff \& Nowak 2004; Markoff et al. 2005): these authors have computed jet models where the synchro-self Compton or the external Comptonization radiation are the dominant processes generating $\mathrm{X}$-ray spectra in $\mathrm{BH}$ binaries. The high-energy emission seen during the LHS is interpreted as synchrotron, Comptonization and reflection emission from the jet that extends from radio to hard X-rays, naturally explaining the correlations observed during the LHS.

We have accurately studied SWIFT J1753.5-0127 and found that, although clearly in LHS, this source is interestingly well below the radio/X-ray correlation laws of $\mathrm{BHs}$ binaries in LHS determined previously by different authors (Corbel et al. 2003; Gallo et al. 2003, 2006), even assuming a large distance. Another possibility is that SWIFT J1753.5-0127 could radiate at a luminosity higher than $5 \%$ of the Eddington regime (while it stayed in the LHS). In our case, thermal Comptonization models fit relatively well our simultaneous RXTE/INTEGRAL data. However, the source and geometry of Comptonization (torus around the $\mathrm{BH}$ or corona over the disk) are not defined. The emission could also be compatible with the standard picture of synchrotron and inverse Compton radiation coming from a self-absorbed conical jet (Markoff et al. 2005). Its contribution in the IR and optical ranges, which is probably dominated in our case by the emission from a cold thermal disk, is small.

Thanks to our INTEGRAL AO3 proposal triggered simultaneously with RXTE, NTT, REM and VLA, it has been possible to perform multiwavelength observations of the XN SWIFT J1753.5-0127 in the LHS on a wide energy band. With the combination of all high-energy $(3 \mathrm{keV}-1 \mathrm{MeV}$ ) instruments on board RXTE and $I N$ TEGRAL, we have measured crucial spectral and timing parameters on SWIFT J1753.5-0127 during its hard outburst. This gives clues on the $\mathrm{BH}$ nature of this object.
SWIFT J1753.5-0127 could belong to a small subset of BHCs that are only observed in the LHS while in outburst. This subset includes the well-known source XTE J1118+480 (e.g., Hynes et al. 2000, 2003). The classification in the LMXB is obtained from our optical results. Radio data confirm the presence of a partially selfabsorbed conical jet and indicate that its radio luminosity is an order of magnitude lower than the one previously typically observed for a $\mathrm{BH}$ in the LHS. Our results imply that the source could be located at the reasonable distance of $\sim 6 \mathrm{kpc}$.

The detection and study of a large sample of $\mathrm{XN}$ in outburst (and in decay phase) will provide accumulated multiwavelength data on bright sources in order to obtain crucial evolutions of the spectra, therefore improving our understanding of the galactic $\mathrm{BH}$ physics and origin. Thanks to large changes in a short period of time (as usually the case for LMXB), it will thus shed light on the physics, accretion processes and radiation mechanisms at work in the vicinity of $\mathrm{BH}$ binaries.

We thank the INTEGRAL and RXTE mission planners for programming the $\mathrm{ToO}$ observations described in the paper. M.R. acknowledges financial support from the French Space Agency (CNES) and from the Spanish Ministerio de Educación y Ciencia through a Juan de la Cierva fellowship linked to the project AYA2004-07171-C02-01, which is partially supported by FEDER funds. SC thanks the ESO staff and especially Cédric Foellmi for performing these ToO observations. PU and AT has been supported by the Italian Space Agency via the grant I/R/046/04. The present work is based on ESO observations (through part of program \# 075.D-0634) and with the use of INTE$G R A L$, an ESA project with instruments and science data center funded by ESA member states (especially the PI countries: Denmark, France, Germany, Italy, Switzerland, Spain, Czech Republic and Poland, and with the participation of Russia and the USA). This research has made use of the NASA Astrophysics Data System Abstract Service and of the SIMBAD database, operated at the CDS, Strasbourg, France.

\section{REFERENCES}

Arnaud, K. A. 1996 in ASP Conf Series 101, Astronomical Data Analysis Software and Systems V, Eds. Jacoby G. H. \& Barnes J. (San Francisco: ASP), 17

Belloni, T. 2001, in "X-ray Emission from Accretion onto Black Holes", Workshop Proceedings (Baltimore, June 20-23), Eds. T. Yaqoob \& J. H. Krolik

Belloni, T., Psaltis, D. \& van der Klis, M. 2002, ApJ, 572, 392

Belloni, T. 2005, COSPAR Proceedings (January 17-20, Mumbai), astro-ph 0507556

Belloni, T., Homan, J., Casella, P., et al. 2005, A\&A, 440, 207

Blandford, R. D. \& Konigl, A. 1979, ApJ, 232, 34

Bohlin, R. C., Savage, B. D. \& Drake, J. F. 1978, ApJ, 224, 132

Bradshaw, C. F., Fomalont, E. B. \& Geldzahler, B. J. 1999, ApJ, 512, L121

Brocksopp, C., Fender, R. P., Larionov, V., et al. 1999, MNRAS, 309, 1063

Brocksopp, C., Jonker, P. G., Fender, R. P., et al. 2001, MNRAS, 323,517

Burrows, D. N., Racusin, J., Morris, D. C., et al. 2005, ATEL 547

Cadolle Bel, M., Rodriguez, J., Sizun, P., et al. 2004, A\&A, 426, 659
Cadolle Bel, M., Rodriguez, J., Goldwurm, A., et al. 2005, ATEL 574

Cadolle Bel, M., Sizun, P., Goldwurm, A., et al. 2006, A\&A, 446, 591

Cardelli, J. A., Clayton, G. C. \& Mathis, J.S. 1989, ApJ, 345, 245

Chaty, S. 2006, VI Microquasar Workshop Proceedings (18-25 September, Como), Ed. T. Belloni

Chaty, S. \& Bessolaz, N. 2006, A\&A, 455, 639

Chaty, S., Haswell, C. A., Malzac, J., et al. 2003, MNRAS, 346, 689

Chen, W., Shrader, C. R. \& Livio, M. 1997, ApJ, 491, 312

Chincarini, G., Zerbi, F. M., Antonelli, A. et al. 2003, The Messenger 113, 40

Corbel, S., Fender, R. P., Tzioumis, A. K., et al. 2000, A\&A, 359, 251

Corbel, S., Nowak, M. A., Fender, R. P., et al. 2003, A\&A, 400, 1007

Corbel, S., Fender, R. P., Tomsick, J. A., et al. 2004, ApJ, 617, 1272

Covino, S., Stefanon, M. Fernandez-Soto, A. et al. 2004, SPIE 5492, 1613

Denis, M., Olive, J.-F., Mandrou, P., et al. 1994, ApJ, 92, 459

Dickey, J. M. \& Lockman, F. J. 1990, ARA\&A, 28, 215 
Done, C., Mulchaey, J. S., Mushotzky, R. F., et al. 1992, ApJ, 395, 275

Fender, R. P., Belloni, T., \& Gallo, E. 2004, MNRAS, 355, 1105

Fender, R., Garrington, S. \& Muxlow, T. 2005, ATEL 558

Fender, R. P., Belloni, T., \& Gallo, E. 2006, in "From X-ray Binaries to Quasars": Black Hole Accretion on All Mass Scales, Eds. T. J. Maccarone, R. P. Fender, and L. C. Ho (Dordrecht: Kluwer)

Finoguenov, A. V., Gilfanov, M. P., Churazov, E. M., et al. 1996, Astronomy Letters, 22, 72

Fuchs, Y., Rodriguez, J., Mirabel, I. F., et al. 2003, A\&A, 409, L35

Gallo, E., Fender, R. P., \& Pooley, G. G., 2003, MNRAS, 344, 60

Gallo, E., Fender, R. P., Miller-Jones, J. C. A., et al. 2006, MNRAS, 370,1351

Gelino, D. M., Balman, S., Kiziloglu, Ü., et al. 2006, ApJ, 642, 438

Gierliński, M., Zdziarski, A. A., Done, C., et al. 1997, MNRAS, 288,958

Gierliński, M., Zdziarski, A. A, Poutanen, J., et al. 1999, MNRAS, 309,496

Gleissner, T., Wilms, J., Pooley, G. G., et al. 2004, A\&A, 425, 1061

Goldwurm, A., David, P., Foschini, L., et al. 2003, A\&A, 411, L223

Gros, A., Goldwurm, A., Cadolle Bel, M., et al. 2003, A\&A, 411, L179

Grove, J. E., Johnson, W. N., Kroeger, R. A., et al. 1998, ApJ, 500, 899

Halpern, J. P. 2005, ATEL 549

Homan, J., Wijnands, R., van der Klis, M., et al. 2001, ApJS, 132, 377

Hynes, R. I., C. W. Mauche, Haswell, C. A., et al. 2000, ApJ, 539, 37

Hynes, R. I., Haswell, C. A., Cui, W., et al. 2003, MNRAS, 345, 292

Hynes, R. I., Robinson, E. L. \& Bitner, M. 2005, ApJ, 630, 405

Jensen, P.-L., Clausen, K., Cassi, C., et al. 2003, A\&A, L7

Kalemci, E., Tomsick, J., Rothschild, J. A., et al. 2001, ApJ, 563, 239

Kalemci, E., 2002, Bulletin of the American Astronomical Society, 201,5705

Landolt, A. U. 1992, AJ, 104, 340

Leahy, D. A., Darbro, W., Elsner, R. F., et al. 1983, ApJ, 266, 160

Maccarone, T. J. 2003, A\&A, 409, 697

Magdziarz, P., \& Zdziarski, A. A. 1995, MNRAS, 273, 837

Markoff, S. \& Nowak, M. A. 2004, ApJ, 609, 972

Markoff, S., Nowak, M. A. \& Wilms, J. 2005, ApJ, 635, 1203

Martí, J., Paredes, J. M. \& Ribó, M. 1998, A\&A, 338, L71

Mathis, J. S. \& Cardelli, J. A. 1992, ApJ, 398, 610

McClintock, J. E. \& Remillard, R. A. 2006, in "Compact Stellar X-ray Sources", Eds. W. H. G. Lewin \& M. van der Klis
Miller, J. M., Homan, J., \& Miniutti, G. 2006, ApJ, in press, astro-ph 0605190

Mitsuda, K., Inoue, H., Koyama, K., et al. 1984, PASJ, 36, 741

Monet, D. G., Levine, S. E., Canzian, B., et al. 2003, AJ, 125, 984 (http://cdsweb.u-strasbg.fr/viz-bin/Cat?I/284)

Morgan, E., Swank, J., Markwardt, C., et al. 2005, ATEL 550

Morris, D. C., Burrows, D. N., Racusin, J., et al. 2005, ATEL 552

Munari, U. \& Zwitter, T. 1997, A\&A, 318, 269

Nowak, M. A., Wilms, J., Heinz, S., et al. 2005, ApJ, 626, 1006

Palmer, D. M., Barthelmey, S. D., Cummings, J. R., et al. 2005 , ATEL 546

Predehl, P., \& Schmitt, J. H. M. M. 1995, A\&A, 293, 889

Ramadevi, M.C. \& Seetha, S. 2006, submitted to MNRAS, astro-ph 0611375

Remillard, K., Garcia, M., Torres, M. A. P., et al. 2005, ATEL 384

Ribó, M. 2005, ASPC, 340, 269

Rodriguez, J., Varnière, P., Tagger, M. , et al. 2002, A\&A, 387, 487

Rodriguez, J., Corbel, S. \& Tomsick, J. A. 2003, ApJ, 595, 1032

Rodriguez, J., Corbel, S., Kalemci, E., et al. 2004a, ApJ, 612, 1018

Rodriguez, J., Corbel, S., Hannikainen, D., et al. 2004b, ApJ, 615, 416

Rodriguez, J., Shaw, S. E. \& Corbel, S. 2006a, A\&A, 451, 1045

Rodriguez, J., Cadolle Bel, M., Tomsick, J. A., et al. 2006b, submitted to ApJ, astro-ph 0611341

Roques, J.-P., Bouchet, L., Jourdain, E., et al. 1994, ApJ, 92, 451

Ruelas-Mayorga, R. A. 1991, Rev. Mex. Astron. Astrofis., 22, 27

Russell, D. M., Fender, R. P., Hynes, R.I., et al. . 2006, MNRAS, 371,1334

Schlegel, D. J., Finkbeiner, D. P., \& Davis, M. 1998, ApJ, 500, 525

Shaposhnikov, N. \& Titarchuk, L. 2006, ApJ, 643, 1098

Skinner, G., \& Connell, P. 2003, A\&A, 411, L123-126

Sobczak, G. J., McClintock, J. E., \& Remillard, R. R. 1999, ApJ, 520,776

Sobczak, G. J., McClintock, J. E. , \& Remillard, R. R. 2000, ApJ,544, 993

Still, M., Roming, P., Brocksopp, C., et al. 2005, ATEL 553

Stirling, A., Spencer, R. E., de la force, C. J., et al. 2001, MNRAS, 327,1273

Tanaka, Y., \& Shibazaki, N. 1996, ARA\&A, 34, 607

Titarchuk, L. 1994, ApJ, 434, 570

Torres, M. A. P., Steeghs, D., Garcia, M. R., et al. 2005a, ATEL 551

Torres, M. A. P., Steeghs, D., Blake, C., et al. 2005b, ATEL 566

Westergaard, N. J., Kretschmar, P., Oxborrow, C. A., et al. 2003, A\&A, 411, L257

Zdziarski, A. A., Grove, J. E., Poutanen, J., et al. 2001, ApJ, 554, L45

Zerbi, F. M., Chincarini, G., Ghisellini, G. et al. 2001, AN 322, 275 
TABLE 1

LOG OF THE SWIFT J1753.5-0127 OBSERVATIONS ANALYZED IN THIS PAPER.

\begin{tabular}{cllll}
\hline Spacecraft & Instrument & $\begin{array}{l}\text { Observation Period } \\
\text { (MJD-53590; U.T.) }\end{array}$ & $\begin{array}{l}\text { Exposure } \\
(\mathrm{ks})\end{array}$ & $\begin{array}{l}\text { Observation } \\
\text { Type/Mode }\end{array}$ \\
\hline INTEGRAL & JEM-X & $2.52-4.64 ; 12: 29-15: 31^{a}$ & 170 & ToO, HEX ${ }^{b}$ \\
INTEGRAL & IBIS & $2.52-4.64 ; 12: 29-15: 31^{a}$ & 176 & ToO, HEX $^{b}$ \\
INTEGRAL & SPI & $2.52-4.64 ; 12: 29-15: 31^{a}$ & 134 & ToO, HEX ${ }^{b}$ \\
Rossi-XTE & PCA & $3.20 ; 4: 51^{c}$ & 3.1 & Public \\
Rossi-XTE $^{c}$ & HEXTE & $3.20 ; 4: 51^{c}$ & 2.2 & Public \\
- & NTT/EMMI & $3.08^{c} ; 02: 07-05: 42$ & 3.6 & ToO \\
- & REM/ROSS ${ }^{d}$ & $3.98 ; 11.98^{e}$ & 1 & ToO \\
- & REM/REMIR & $3.03 ; 11.03^{e}$ & 7.3 & ToO \\
- & VLA & $3.19-3.35 ; 04: 20-08: 50$ & 16.2 & ToO \\
\hline
\end{tabular}

Notes:

a) Plus 2 days;

b) Hexagonal pattern around the nominal target location;

c) At the beginning of the observation;

d) 0.6 ks observations also taken on 53599.99 (U.T. 17.99) and 53640.04 MJD (U.T. 27.04) respectively (see Section 3.5);

e) At mid-observations. 
TABle 2

Best-fit parameters of individual components used to fit the Power Density Spectrum (errors at $90 \%$ CONFIDENCE LEVEL: $\left.\Delta \chi^{2}=2.7\right)$.

\begin{tabular}{llll}
\hline Lorentzian \# & $\begin{array}{l}\text { Centroid Frequency } \\
(\mathrm{Hz})\end{array}$ & $\begin{array}{l}\text { Width } \\
(\mathrm{Hz})\end{array}$ & $\begin{array}{l}\text { RMS amplitude } \\
(\%)\end{array}$ \\
\hline $\mathrm{n} 1$ & 0 (Frozen) & $0.92_{-0.06}^{+0.05}$ & $26.8_{-1.9}^{+1.6}$ \\
$\mathrm{n} 2$ & 0 (Frozen) & $9.9_{-1.3}^{+1.4}$ & $16.8_{-2.6}^{+2.9}$ \\
$\mathrm{QPO}$ & $0.241 \pm 0.006$ & $0.03_{-0.01}^{+0.02}$ & $5.4_{-1.9}^{+1.7}$ \\
\hline
\end{tabular}


TABLE 3

SUMMARY OF RADIO OBSERVATIONS.

\begin{tabular}{lrrrr}
\hline $\begin{array}{l}S_{1.4 \mathrm{GHz}} \\
(\mathrm{mJy})\end{array}$ & $\begin{array}{l}S_{4.9 \mathrm{GHz}} \\
(\mathrm{mJy})\end{array}$ & $\begin{array}{l}S_{8.5 \mathrm{GHz}} \\
(\mathrm{mJy})\end{array}$ & $\begin{array}{l}S_{15 \mathrm{GHz}} \\
(\mathrm{mJy})\end{array}$ & $\alpha_{1.4-8.5 \mathrm{GHz}}$ \\
\hline $0.65 \pm 0.15$ & $0.65 \pm 0.07$ & $0.7 \pm 0.1$ & $<0.60$ & $+0.03 \pm 0.03$ \\
$(0.04)$ & $(0.04)$ & $(0.01)$ & $(0.13)$ & \\
\hline
\end{tabular}

Note: Errors are at the $1 \sigma$ level and a $3 \sigma$ upper limit is reported at $15 \mathrm{GHz}$. The expected r.m.s. sensitivities are given in parentheses. 
TABLE 4

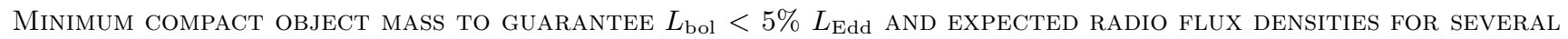
Distances to SWIFT J1753.5-0127 (aCCORDing to the RELATion of Gallo Et Al. 2003, SEe text).

\begin{tabular}{lcc}
\hline $\begin{array}{l}d \\
(\mathrm{kpc})\end{array}$ & $\begin{array}{c}\text { Minimum compact } \\
\text { object mass }\left(\mathrm{M}_{\odot}\right)\end{array}$ & $\begin{array}{c}S_{5} \mathrm{GHz} \\
(\mathrm{mJy})\end{array}$ \\
\hline 1 & 0.3 & $39.71 \pm 27.78$ \\
2 & 1.0 & $26.20 \pm 18.33$ \\
3 & 2.2 & $20.54 \pm 14.37$ \\
4 & 4.0 & $17.28 \pm 12.09$ \\
5 & 6.2 & $15.12 \pm 10.58$ \\
6 & 8.9 & $13.55 \pm 9.48$ \\
7 & 12.1 & $12.35 \pm 8.64$ \\
8 & 15.8 & $11.40 \pm 7.98$ \\
9 & 20.0 & $10.63 \pm 7.43$ \\
10 & 24.7 & $9.97 \pm 6.98$ \\
11 & 29.9 & $9.42 \pm 6.59$ \\
12 & 35.5 & $8.94 \pm 6.25$ \\
13 & 41.7 & $8.52 \pm 5.96$ \\
14 & 48.4 & $8.15 \pm 5.70$ \\
15 & 55.5 & $7.82 \pm 5.47$ \\
\hline
\end{tabular}

Note: Errors at the $1 \sigma$ level. 


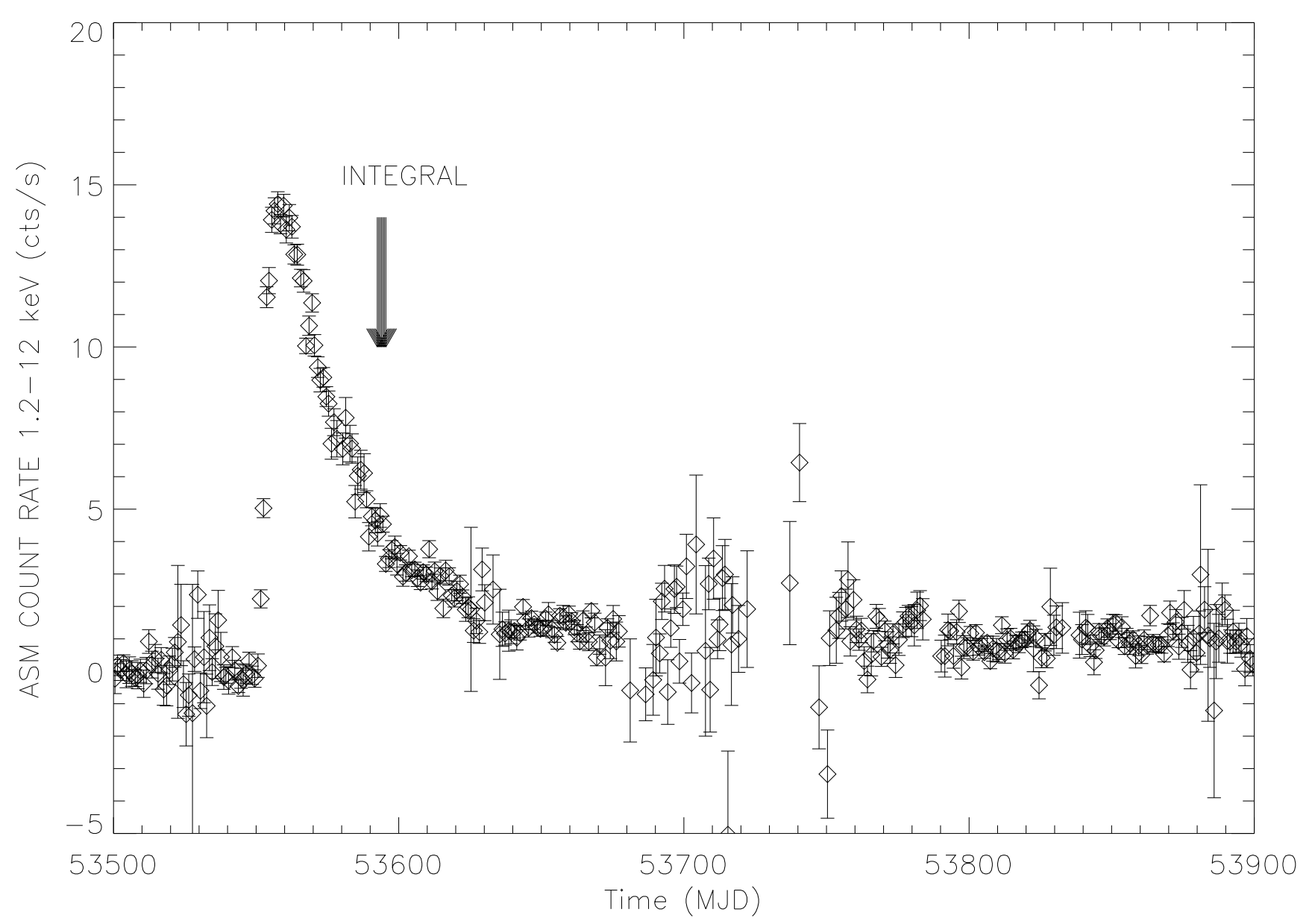

Fig. 1. - RXTE/ASM daily average (1.2-12 keV) light curve of SWIFT J1753.5-0127 from 2005 mid-May up to 2006 mid-June (MJD = JD - 2400000.5) with the periods of our simultaneous INTEGRAL observations indicated by an arrow (its width illustrates the INTEGRAL range). The gap corresponds to the passage of the Sun close to the source. Error bars are at $90 \%$ confidence level. 


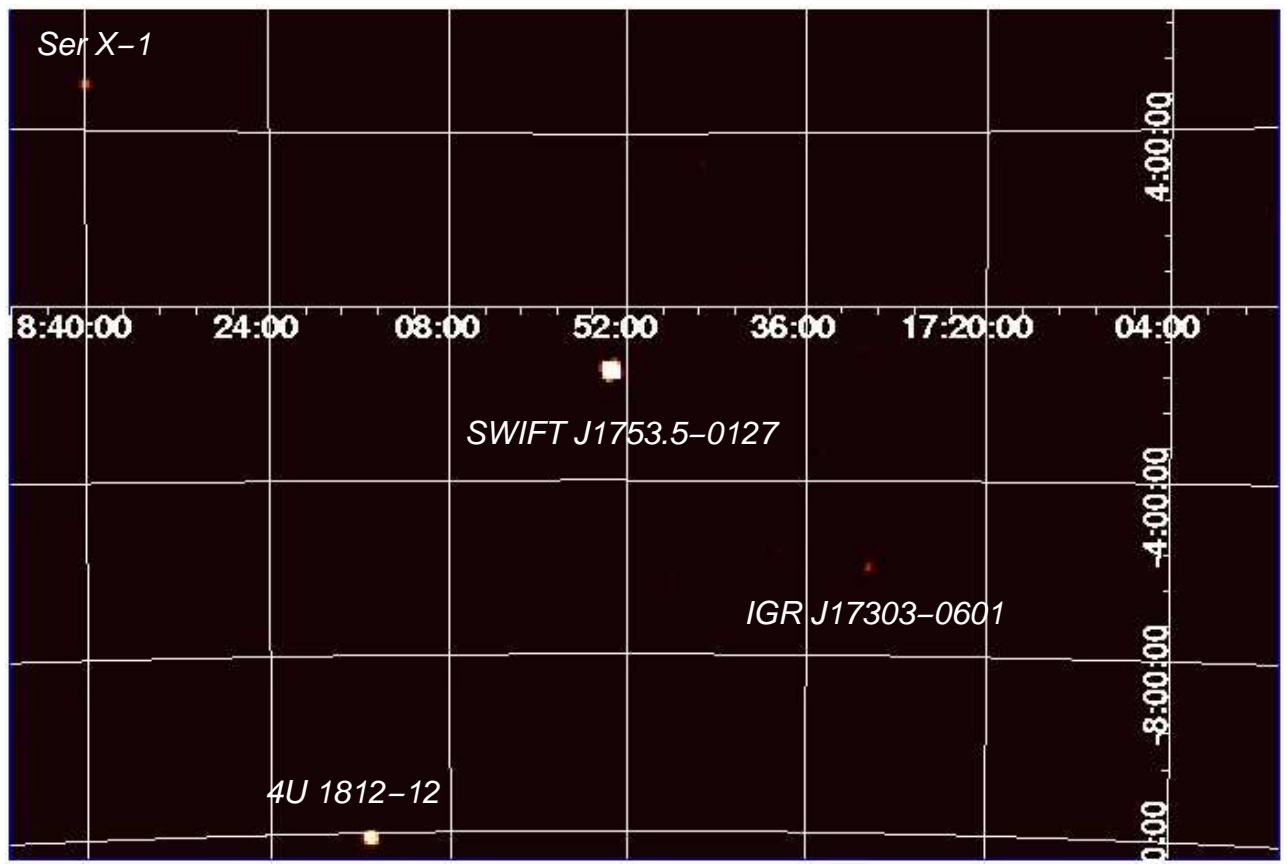

FIG. 2.- The IBIS/ISGRI reconstructed sky image of the region around SWIFT J1753.5-0127 in the $20-40$ keV band. SWIFT J1753.5-0127 appears at a significance level of $440 \sigma$ over the background. The other sources in the image are the neutron star $4 \mathrm{U} 1812-12($ at $34 \sigma)$, the X-ray burster Ser X-1 $(14 \sigma)$ and the INTEGRAL obscured source IGR J17303-0601 (12 $\sigma)$. 


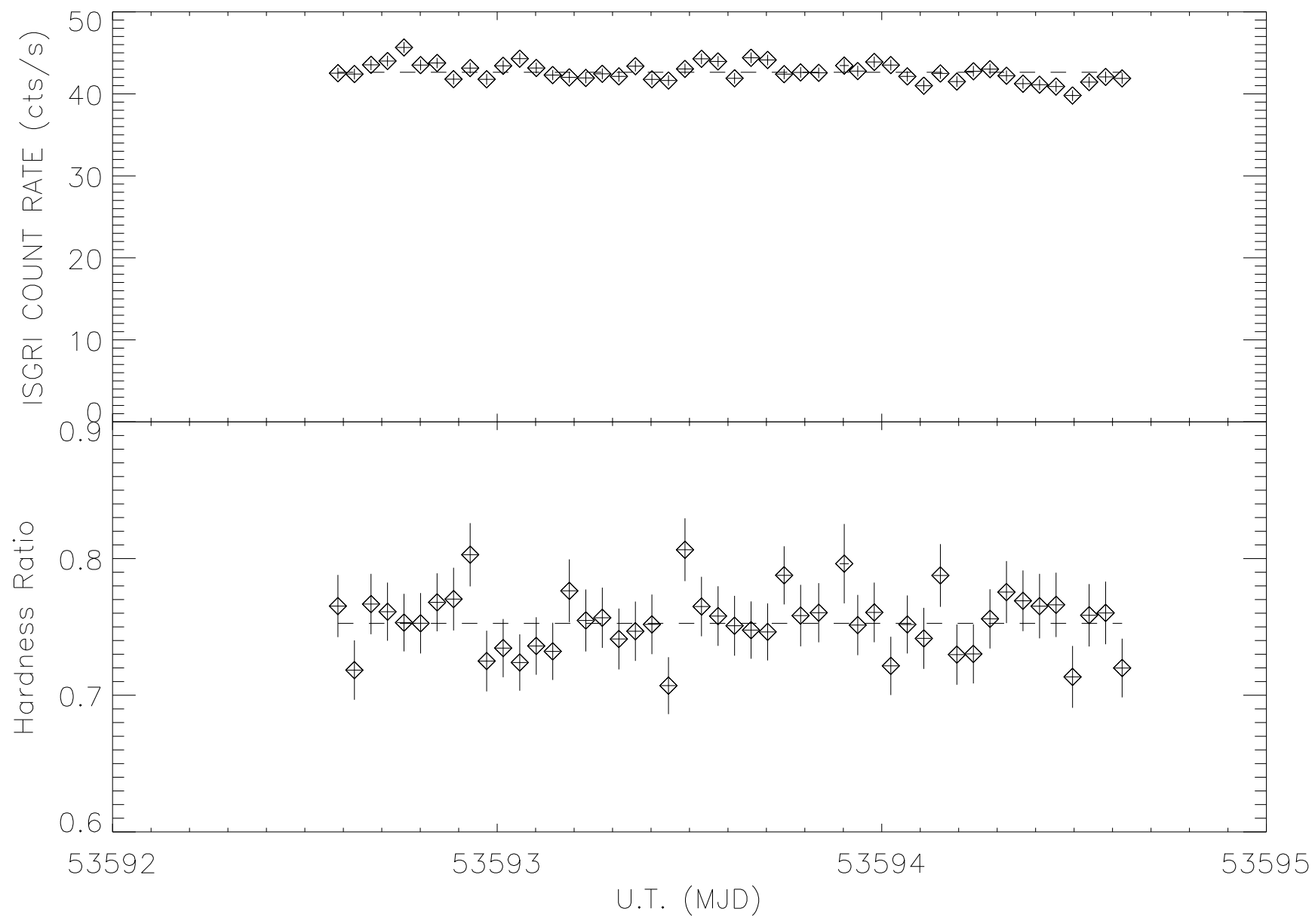

FIG. 3. - The 20-320 keV IBIS/ISGRI light curve of SWIFT J1753.5-0127 and corresponding HR between the 40-80 and the 20-40 keV significant energy bands (average levels denoted by dashed line) during our ToO INTEGRAL observations. Error bars are at $90 \%$ confidence level. 


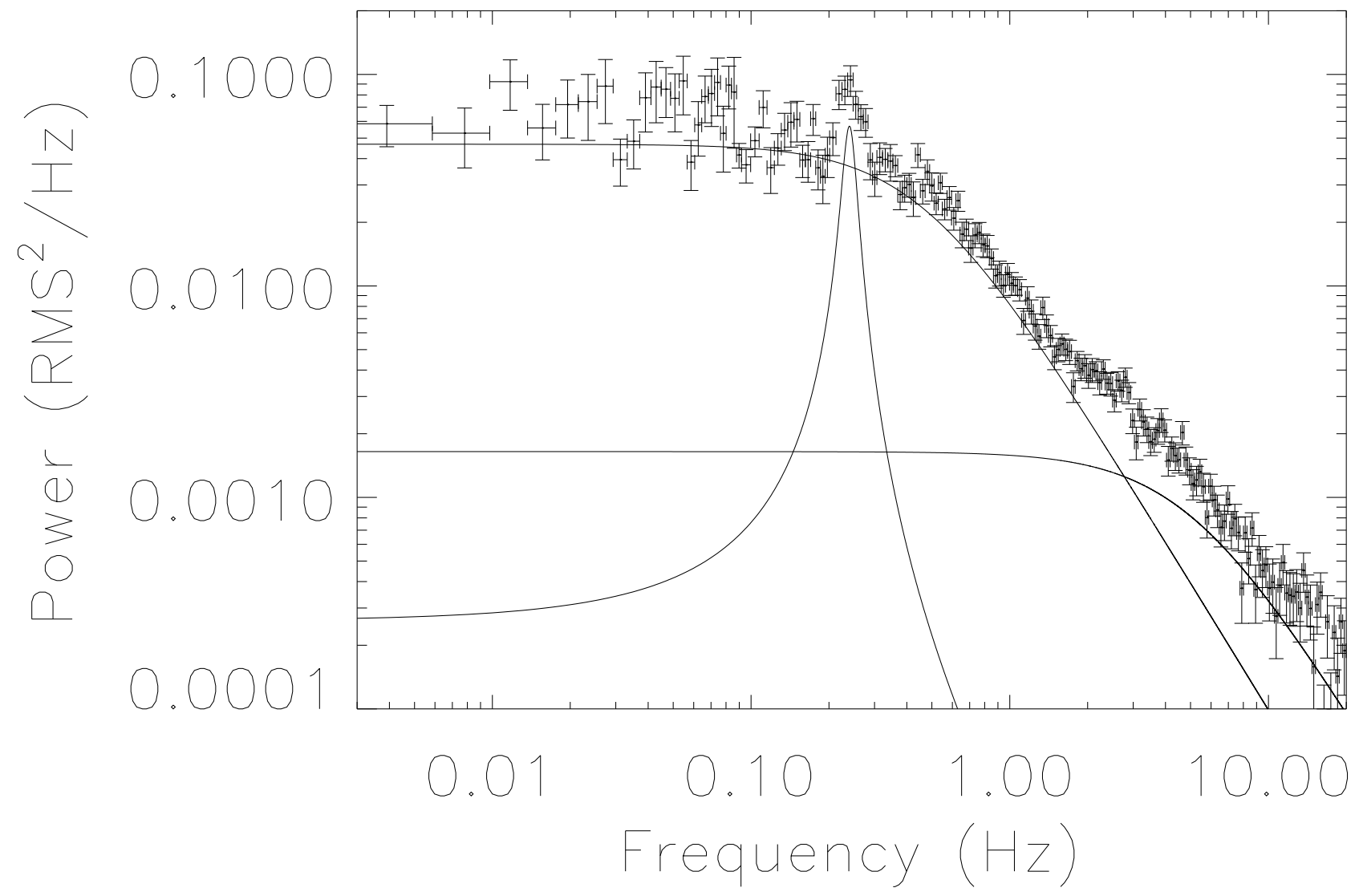

FIG. 4.- Power Density Spectrum of SWIFT J1753.5-0127 obtained with the RXTE/PCA data on 2005 August 11 . A QPO frequency of $0.24 \mathrm{~Hz}$ is found; an F-test confirms its necessity at the $99.95 \%$ level. The lines correspond to the three Lorentzians which are necessary to best fit the data (see Table 21): we obtain a $\chi_{\text {red }}^{2}$ of 1.20 (163 dof). 


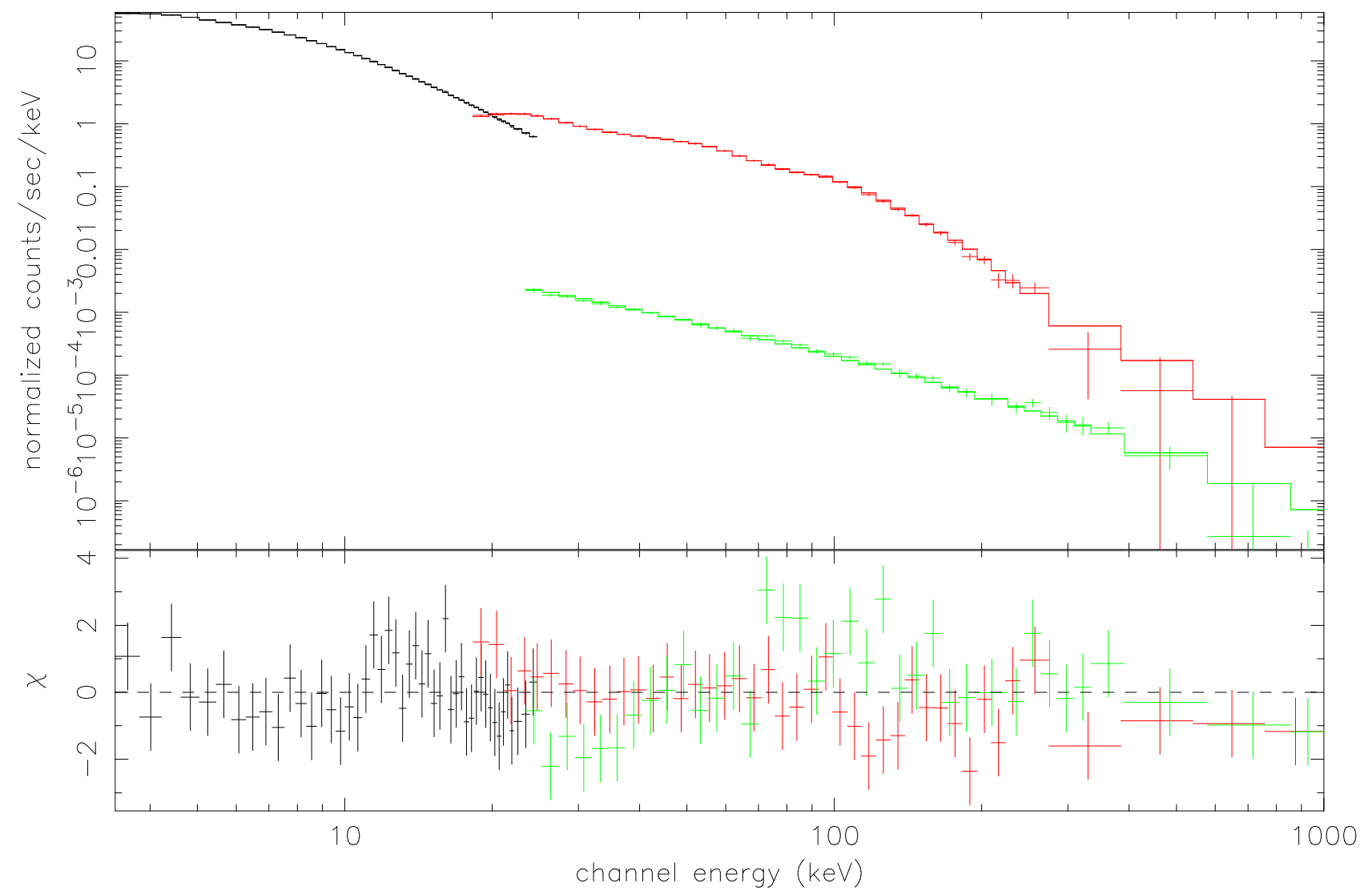

FIG. 5.- Spectra of SWIFT J1753.5-0127 during our INTEGRAL ToO with the PCA (black), IBIS (red) and SPI (green) data along with the best-fit model: absorbed thermal Comptonization convolved by reflection (see text). Residuals in $\sigma$ units are also shown. 


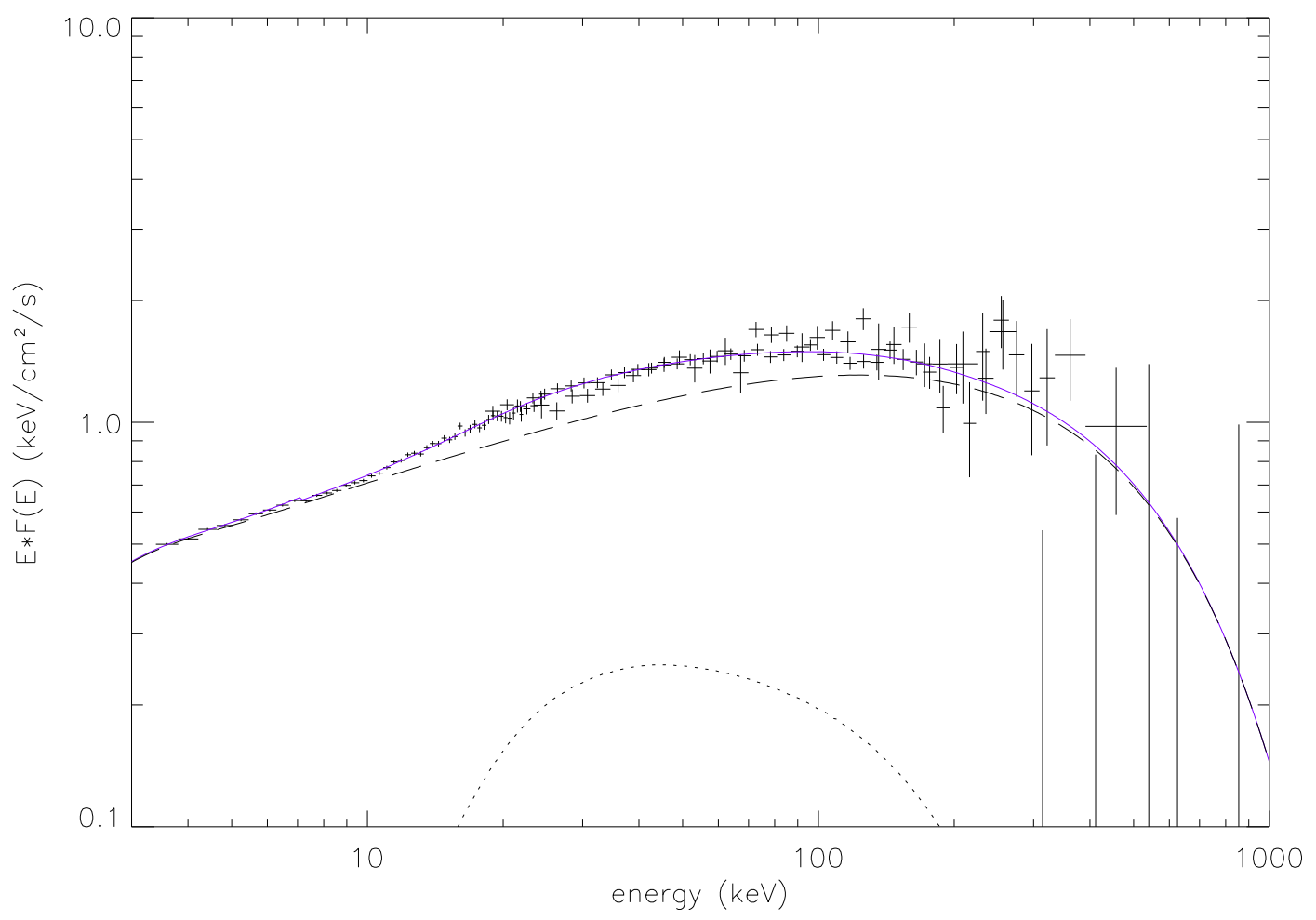

FIG. 6.- $E F(E)$ spectra (units $\mathrm{keV} \mathrm{cm}^{-2} \mathrm{~s}^{-1}$ ) of SWIFT J1753.5-0127 during our INTEGRAL ToO with the PCA, IBIS and SPI data along with the best-fit model (thick): absorbed Comptonization (dashed) convolved by reflection (dotted). 


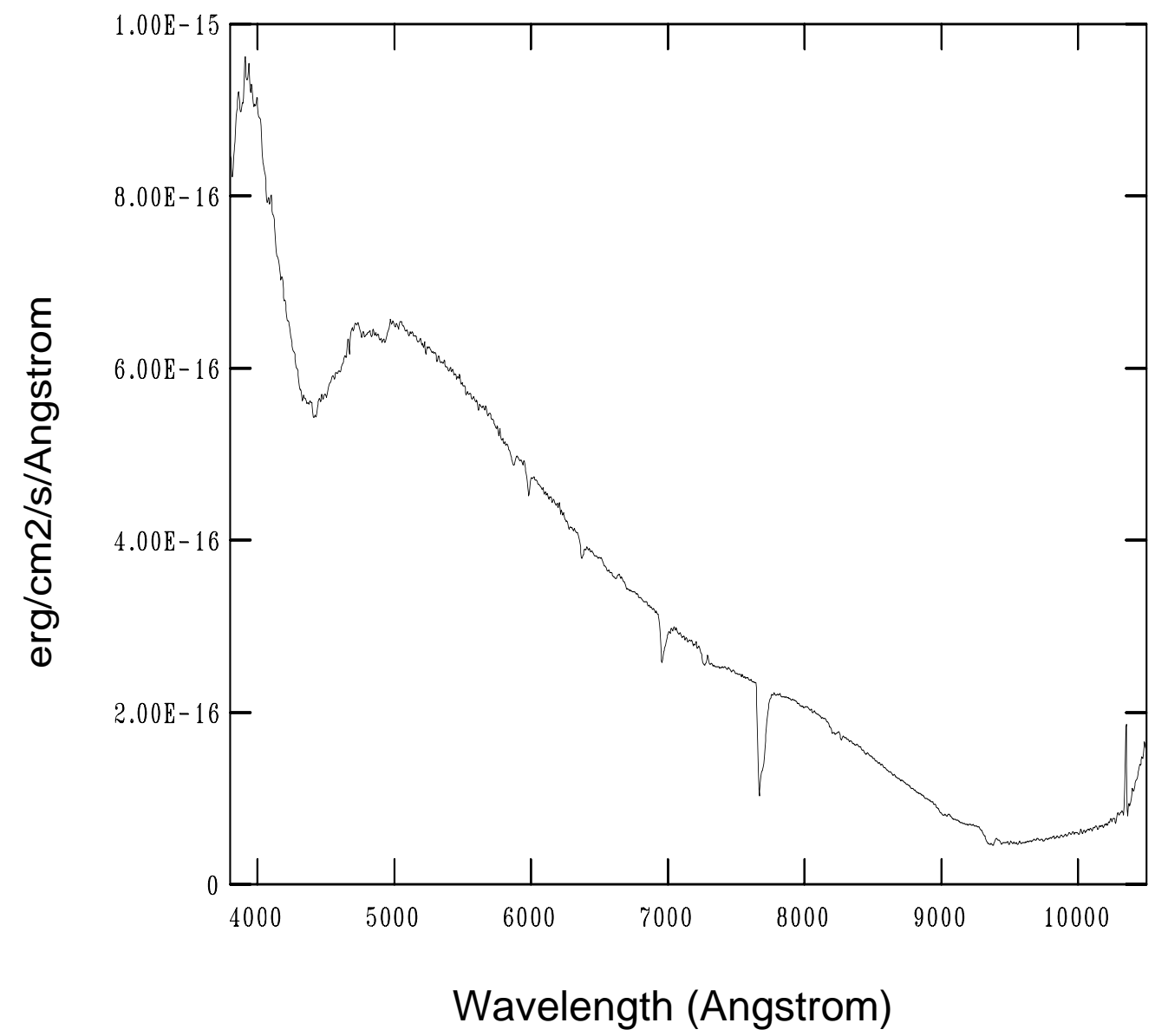

FIG. 7.- Calibrated optical spectrum of SWIFT J1753.5-0127 (erg $\mathrm{cm}^{-2} \mathrm{~s}^{-1} \AA^{-1}$ ) obtained on 2005 August 11 around U.T. 02 (see Table 1 for accurate dates) with the NTT telescope (see text for line details). 


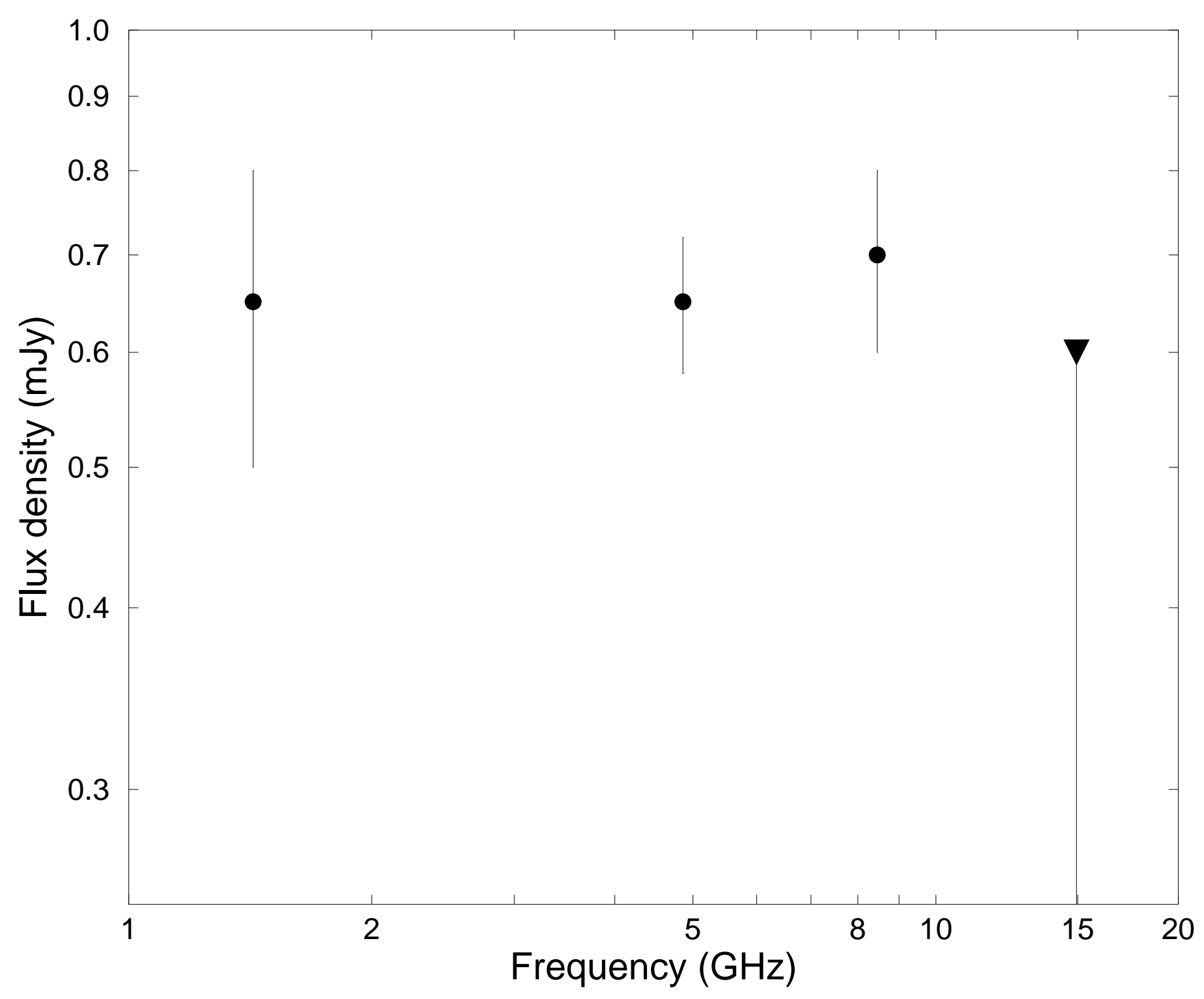

FIG. 8. - Radio spectrum of SWIFT J1753.5-0127 from 1.4 to $15 \mathrm{GHz}$. Error bars are at the $1 \sigma$ level while we show a $3 \sigma$ upper limit at $15 \mathrm{GHz}$. The data are compatible with a flat radio spectrum, interpreted as synchrotron radiation from a partially self-absorbed conical jet. 


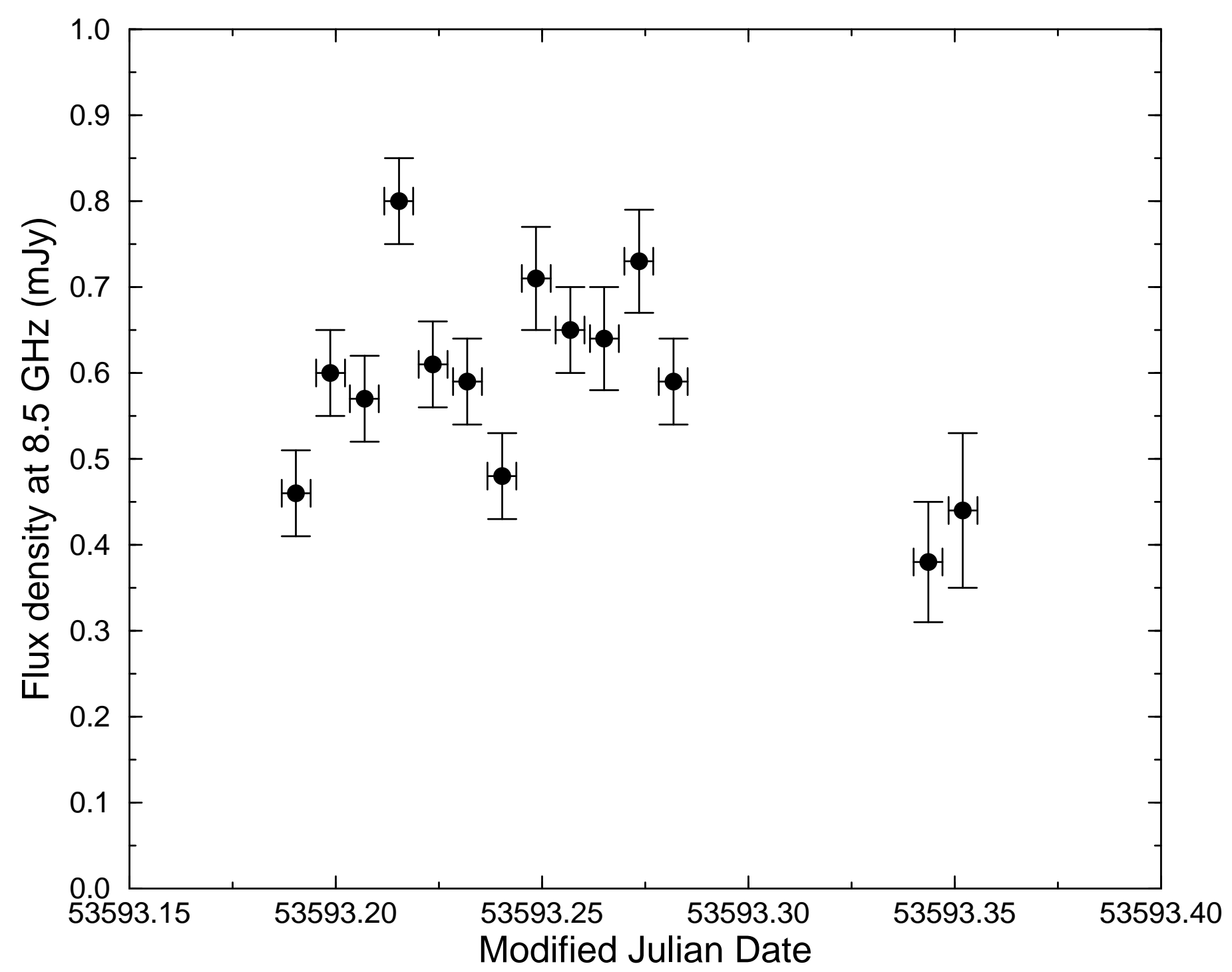

FIG. 9. - Radio lighcurve of SWIFT J1753.5-0127 obtained with the VLA at $8.5 \mathrm{GHz}$. The final two data points were acquired at low elevations and could have flux calibration errors higher than those shown. Even if excluding them, a $\chi^{2}$ test reveals that the data are not compatible with a constant radio flux density within the $99.5 \%$ confidence level. 


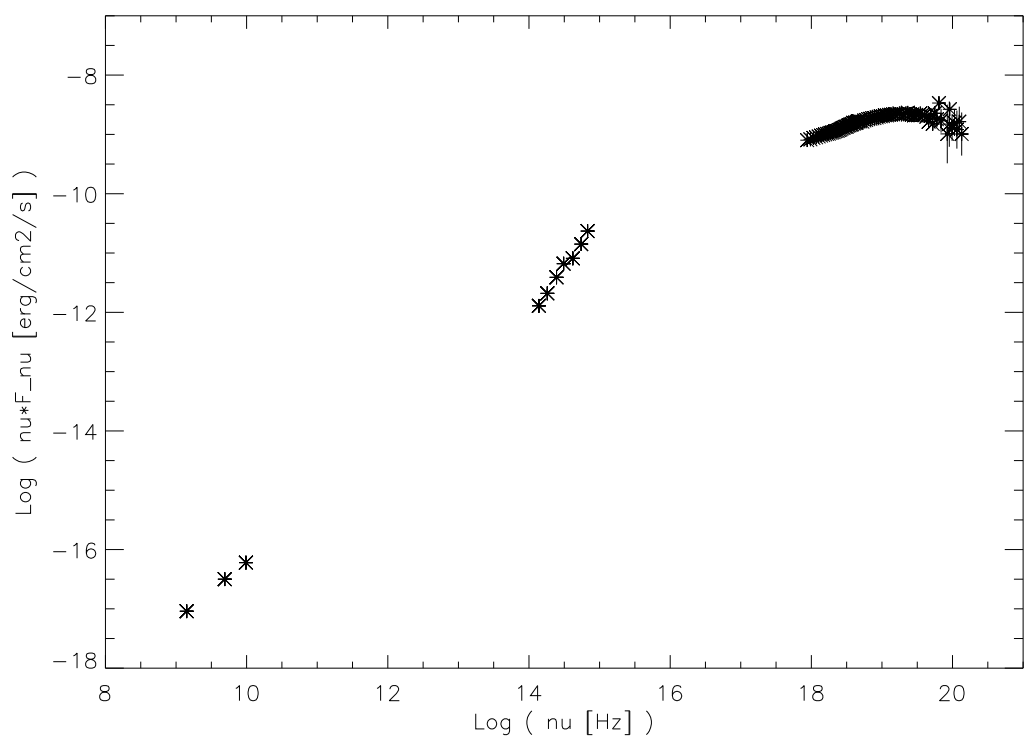

FIG. 10. - Spectral energy distribution of SWIFT J1753.5-0127 on August 11 with radio (15 GHz upper limit data point excluded), IR, optical and X-ray points (up to $535 \mathrm{keV}$ ) and errors. The NIR and optical flux densities (filters $B, V, R, I, J, H$ and $K$ ) were dereddened with $A_{V}=1.05 \pm 0.12 \mathrm{mag}$ (see text). A simple power law can not fit all the points together: there are at least three disctinct contributions (the optically thick synchrotron emission from the jet, the thermal disk and Comptonization of soft photons by a hot medium). 


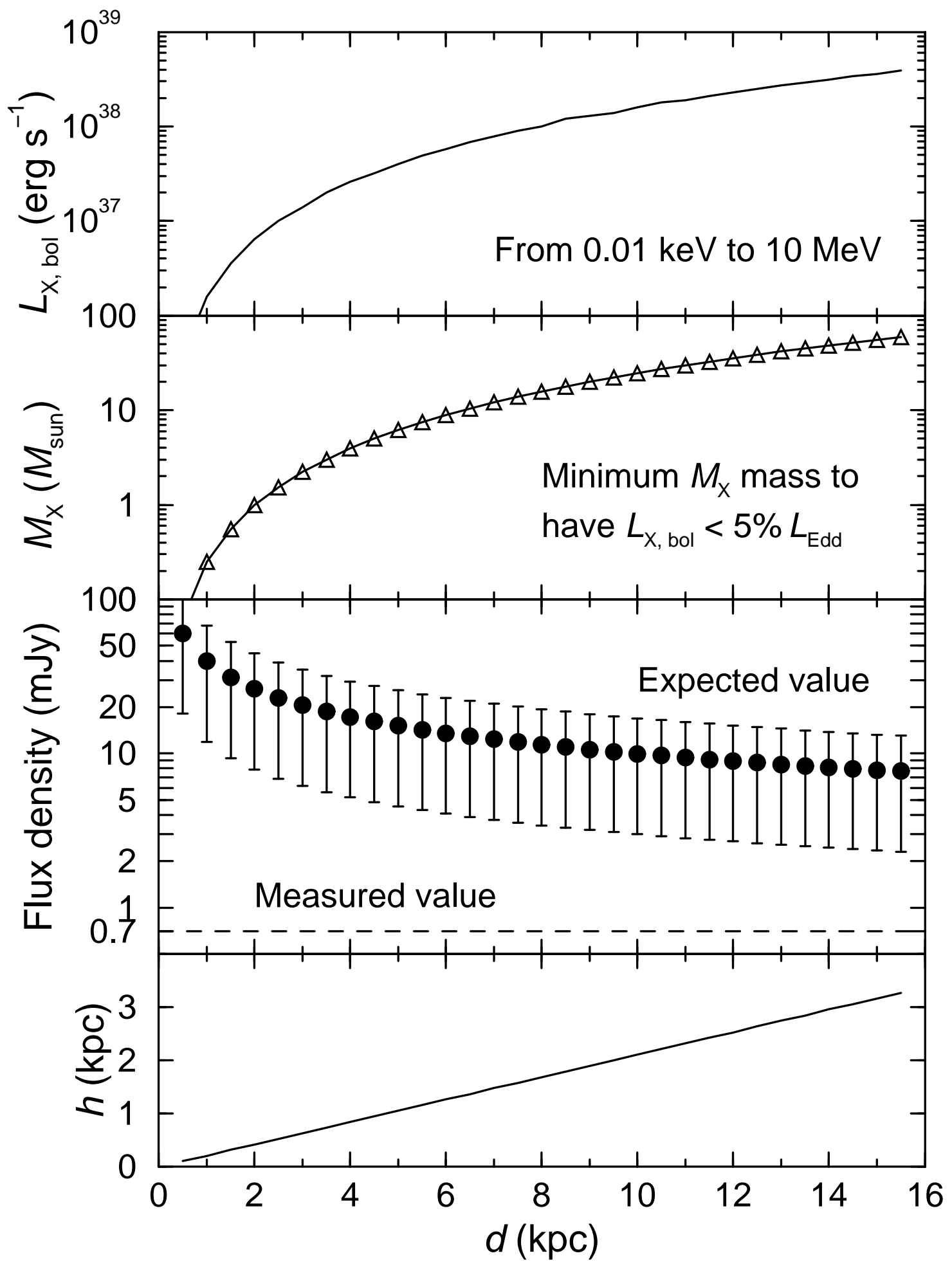

FIG. 11. - From top to bottom as a function of the distance: bolometric unabsorbed luminosity in the $0.01 \mathrm{keV}-1 \mathrm{MeV}$ band; minimum compact object mass to guarantee $L_{\mathrm{bol}}<5 \% L_{\mathrm{Edd}}$; predicted radio flux densities of SWIFT J1753.5-0127 (filled circles with $1 \sigma$ error bars) by using our measured X-ray flux and the correlation found for BH in the LHS (Gallo et al. 2003) and our measured radio flux density at 8.5 $\mathrm{GHz}$ (dashed line) showing a discrepancy of one order of magnitude even for large distances; and height above the galactic plane. 